\title{
DISCRIMINATION IN A MODEL OF CONTESTS WITH INCOMPLETE INFORMATION ABOUT
}

\author{
ABILITY* \\ BY DAVID PÉREZ-CASTRILLO AND DAVID WETTSTEIN ${ }^{\dagger}$ \\ Universitat Autònoma de Barcelona and Barcelona GSE, Spain; Ben-Gurion University \\ of the Negev, Israel
}

March 22, 2015

\begin{abstract}
We study contests with private information and identical contestants, where contestants' efforts and innate abilities generate output of varying qualities. The designer's revenue depends on the quality of the output and she offers a reward to the
\end{abstract}

* Manuscript received May 2014; revised December 2014.

†This paper was previously circulated as the working paper "Innovation contests." We thank Matthias Dahm, Todd Kaplan, Diego Moreno, Aner Sela, Ron Siegel and Charles Zheng for useful discussions and comments, as well as participants in the 22nd International Conference on Game Theory in Stony Brook, the Contests, Mechanisms \& Experiments Conference in Exeter, the 3rd York Annual Symposium in Game Theory, the M-SWET Conference in Madrid, GAMES 2012 in Istanbul, ASSET meeting in Limassol, BGSE Winter Workshop, 2012 China International Conference on Game Theory, and seminars in Shanghai University of Finance and Economics, Paris School of Economics, Universitat de Barcelona and Universitat Rovira i Virgili. We also thank the Editor and three reviewers for their insightful comments. We gratefully acknowledge financial support of the Ministerio de Ciencia y Tecnología ECO2009-07616 and ECO2012-31962, Generalitat de Catalunya (2014SGR-142), the Severo Ochoa Programmme (SEV2011-0075), the Pinhas Sapir Center for Development in Tel Aviv University, the Israeli Science Foundation (grant No. 1210/12) and ICREA. Pérez-Castrillo is a fellow of MOVE. Please address correspondence to: David Wettstein, Economics Department, Ben-Gurion University of the Negev, Beer-Sheva, Israel. E-mail: wettstn@bgu.ac.il. 
contestant achieving the highest quality. We characterize the equilibrium behavior, outcomes and payoffs for both nondiscriminatory and discriminatory (where the reward is contestant-dependent) contests. We derive conditions under which the designer obtains a larger payoff when using a discriminatory contest and describe settings where these conditions are satisfied. 


\section{Introduction}

Contests are organized for a variety of reasons. They are used to answer "small" questions such as who will do the catering, or "large" questions such as what is the best way to cope with global warming. Designing a contest is a common way to promote competition in several settings such as, government contracts, procurement decisions, research budgets and promotions.

Often, a contest is designed to address a targeted objective (see, for instance, Scotchmer, 2004). Such contests have been used in the past to obtain innovations in various fields including mathematics, food preservation and maritime navigation. Today, they are frequently used by organizations such as the Institute for Advanced Architecture of Catalonia and HP "to promote discussion and research ... that can help us to envisage how the city and the habitat of the 21st century will turn out." Similarly, the U.S.-based Knight Foundation has organized contests to elicit digital news experiments that inform and engage communities. As a final example, Newell and Wilson (2005) and Newell (2008) proposed that the U.S. Department of Energy hold contests to resolve specific technical and scientific challenges related to greenhouse gases mitigation.

In this paper, we introduce a new class of contests with private information. It provides a tractable analytical setting to consider a wide range of economic activities, from procurement to lobbying. It allows explicit derivation of the contestants' behavior in a large class of contests with two-sided private information and to analyze the characteristics of the optimal contest for the designer. The model is suitable for the analysis of common day scenarios of competition where both innate ability and effort generate the final outcome. For expositional purposes, we choose the particular setting of an innovation contest to describe the model.

Our framework is the following. A designer wishes to obtain an innovation that can be produced by $n \geq 2$ agents. The quality of the innovation achieved by an agent depends on his ability and the effort devoted to the task. The agents' abilities are independently drawn from the same distribution function and once an agent observes his ability he decides on his effort. Both ability and effort are an agent's private information. Furthermore, the quality of an innovation is not verifiable. However, an independent party can determine the best-quality innovation. The designer sets up a contest whereby the highest-quality 
innovation receives a prize. ${ }^{1}$ A contest is called nondiscriminatory if the prize does not depend on the winner's identity, otherwise it is called discriminatory.

We characterize the equilibrium behavior in a nondiscriminatory contest with $n$ agents for arbitrary distributions of abilities and determine the sets of agent types that exert positive effort and those that exert zero effort. The boundaries of those sets depend on the distribution function of the ability and the prize.

We then consider discriminatory contests where the reward depends on the identity of the winner. We restrict attention in this part of the analysis to the case where $n=2 .^{2}$ The introduction of discrimination leads to qualitatively different equilibrium behavior, as compared to agents' behavior in nondiscriminatory contests. The two agents' equilibrium behavior in discriminatory contests again involves regions with zero and positive effort levels. The main new feature is the discontinuity in the behavior of the agent assigned the larger reward, who moves discretely from zero to a positive effort level. We provide a full characterization of the equilibrium for a large class of distribution functions including convex and concave functions.

We study the designer's payoff in both discriminatory and nondiscriminatory contests with two agents and find conditions under which the use of a discriminatory contest increases the payoff. That is, in our environment, where the only feasible mechanisms are contests, the optimal mechanism may lead to discrimination via rewards. This result is particularly interesting since it is obtained in a framework where agents are ex-ante identical. That is, discrimination introduces some asymmetry into a symmetric model. Discrimination dominates nondiscrimination in some setups because it leads to an increase in the expected efforts of the agents, thereby resulting in larger expected revenues. In particular, we show a class of simple examples where discrimination increases the designer's payoff. We also prove that a discriminatory contest is better if the distribution of the ability types is a convex function, with very low density at zero, and the designer has a high valuation of the quality of the innovation.

\footnotetext{
${ }^{1}$ Contests like those that we analyze, due to their simplicity and popularity, are also prevalent in many situations where quality is verifiable.

${ }^{2}$ The case of $n>2$ agents presents several technical difficulties because the conditions characterizing the equilibrium behavior do not to lead to closed-form solutions for general distribution functions. The case where $n=2$ allows to highlight the effect discrimination via rewards might have and, in particular, the possibility that it might increase the designer's profits.
} 
Contests in complete information environments have been extensively analyzed. Baye et al. (1996) studied the contestants' equilibrium behavior in standard all-pay auctions with complete information where agents bid for an object, all bids are paid and the highest bidder receives the object. This model was extended in several dimensions by Kaplan et al. (2003), Gurtler and Krakel (2010), Casas-Arce and Martinez-Jerez (2011), Jönsson and Schmutzler (2013) and Siegel (2014). Siegel (2009) and (2010) provided a general method of calculating equilibrium payoffs in a large class of all-pay auctions. Konrad (2009) provided an excellent survey of equilibrium and optimal design in contests.

In scenarios where agents' valuations are private information, Amann and Leininger (1996) characterized the equilibrium bids for all-pay auctions where valuations are independently drawn from possibly asymmetric distribution functions. Krishna and Morgan (1997) extended the analysis to the case in which the bidders' information regarding valuations is affiliated. Moldovanu and Sela (2001), on the other hand, considered the case where the agents' cost of bidding is private information. Finally, Siegel (2012) provided a constructive characterization of the unique equilibrium of asymmetric two-player all-pay auctions.

Our model differs from the literature discussed above in several ways. First, the designer chooses the size of the reward. Second, the private information, regarding the agents' cost is introduced in a novel and tractable way, with ability and effort being perfect substitutes. We can interpret agents' abilities as their head starts in a contest. In this sense, our model is related to Seel (2014), who studied a two-agent all-pay auction model with a common prize in which one of the agents has a private head start. The equilibrium behavior of the informed agent in Seel's (2014) model bears similarities with the contestants' equilibrium behavior in our nondiscriminatory contests. ${ }^{3}$

Singh and Wittman (2001) analyzed a model where quality is a function of both agents' effort and type, and applied the revelation principle to characterize the structure of an optimal nondiscriminatory reward structure. While the environment they considered is quite general, their assumptions exclude the simpler model studied in our paper. Furthermore, their mechanism design analysis is appropriate only for environments where quality

\footnotetext{
${ }^{3}$ In Seel (2014), a discontinuity in the behavior of the agent with private information (agent 1) arises when this agent is disadvantaged. In his framework, agent 2 follows a mixed strategy whereas agent 1 bids zero when he is very weak and jumps to a positive bid if the disadvantage is small enough.
} 
is verifiable, as the quality variable features prominently in the rules of the mechanism. In our environment, qualities can only be relatively evaluated.

Discrimination in our model is via asymmetric rewards in that the designer can offer different prizes among contestants. While the possibility to discriminate among contestants has attracted considerable attention in the literature, to the best of our knowledge only in Gurtler and Krakel (2010) is discrimination not based on the scoring method but rather on the reward structure. Moreover, we study discrimination in a contest where agents are symmetric.

Head starts or handicaps are ways of biasing a contest in favor of (or against) a contestant by translating bids into scores in a way that favors (or handicaps) the contestant relative to his competitors. Baye et al. (1993), Clark and Riis (2000), Che and Gale (2003) and Gurtler and Krakel (2010) showed that it is, in general, beneficial to handicap a stronger contestant so as to level the field when starting from an asymmetric contest. ${ }^{4}$ Our finding that discrimination by offering contestant-specific prizes in an environment with symmetric contestants can increase the designer's payoff runs contrary to those studies. This result is in line with some of the findings in Fu et al. (2012) for complete information settings and Kirkegaard (2012) and Kawamura and Moreno de Barreda (2014) for private information environments regarding discrimination via handicaps or head starts.

Fu et al. (2012) considered $R \& D$ races and analyzed the optimal allocation of a fixed budget with the goal of maximizing the (expected) quality of the winning innovation. In their setting, which is similar to a Tullock contest, they showed that favoring the "weaker" contestant is optimal when $R \& D$ effort is highly effective, whereas it pays to favor the "stronger" contestant when it is not. As regards the prize schedule, they showed that it is actually optimal to offer a uniform prize rather than contestant-specific prizes.

Kirkegaard (2012) analyzed both handicaps and head starts in environments with private valuations where the designer is interested in maximizing total expenditures. For several settings he showed that it pays to strengthen the weaker contestant, but also demonstrated cases where the weaker contestant should be handicapped or where one of

\footnotetext{
${ }^{4}$ Kirkegaard (2013) considered a setup where distribution functions are the same, with several agents that differ in their cost of bidding, and analyzed the effect of handicaps. He showed that some of the supposed beneficiaries may in fact become worse off when the favored group is diverse.
} 
two symmetric contestants should be given a small head start. Kawamura and Moreno de Barreda (2014) considered an all-pay contest where the agents' abilities are common knowledge but are unknown to the designer. They showed that a designer faced with two ex-ante identical contestants, who wishes to identify the contestant with the highest ability, should give a head start to one of them.

Discriminating among identical agents has also been shown to be optimal in models very different from ours. In particular, Levitt (1995) and Winter (2004) found that discriminating is optimal in environments where a principal wants to provide several identical agents with incentives to carry out a task. ${ }^{5}$

The rest of the paper proceeds as follows. In Section 2, we introduce the model. Section 3 analyzes agents' equilibrium behavior in nondiscriminatory contests and Section 4 agents' behavior in discriminatory contests. Section 5 calculates the designer's payoff as a function of the rewards and shows conditions under which it is optimal to discriminate. Section 6 concludes and proposes directions for further research.

\section{The model}

We consider the problem facing an organization that wishes to procure an innovation. ${ }^{6}$ The benefits derived from this innovation depend on its quality $q$ and are given by $\pi(q)$.

There is a set $N$ of $n \geq 2$ identical risk-neutral agents who can realize the desired innovation. The quality of the innovation produced by an agent depends on his type and his choice of nonnegative effort. The agents' types represent their proficiency to develop the particular innovation. Both the types and choices of effort are private information. Denoting the type of agent $i$ by $\theta_{i}$ and his effort by $e_{i}$, the quality of the innovation realized by agent $i$ is given by $q_{i}\left(\theta_{i}, e_{i}\right)=\theta_{i}+e_{i}$.

Agents' types are independently distributed according to the same differentiable and

\footnotetext{
${ }^{5}$ Kräkel (2012) considered contests in order to elicit effort where the contestants have different known abilities. Effort and ability are perfect substitutes in these contests, prizes are given and the designer's decision is whether to hold "fair" contests among identical or "unfair" among non-identical contestants. He showed that unfair contests may be optimal if a weak agent has a higher perceived winner prize when beating a stronger opponent.

${ }^{6}$ As mentioned in the Introduction, we use innovation terminology. However, we could also refer to the procurement of services or commodities.
} 
atomless distribution function $F(\theta)$ on $[0,1]$, with $F^{\prime}(\theta)>0$ for all $\theta \in[0,1]$. Types are revealed to the agents prior to their choice of effort. The qualities of the innovation can be evaluated, only relatively, by an independent authority.

To procure the innovation, the organization holds a contest among the agents. Henceforth, we refer to the organization as the designer of the contest. The winner of the contest is the agent who offers the innovation of the highest quality, with ties broken by having each agent submitting the highest quality win with equal probability. In a nondiscriminatory contest, the designer offers a prize $R$ to the winning agent. The contest is discriminatory when the designer offers different prizes depending on the identity of the winner. In a discriminatory contest, the prize offered to agent $i \in N$, were he to win the contest, is $R_{i}$ and $R_{j} \neq R_{j^{\prime}}$ for some $j, j^{\prime} \in N$.

Given a contest, agents simultaneously choose their effort. In a nondiscriminatory contest, the payoff of any agent when he chooses effort $e$ is $R-e$ in case he wins the contest and $-e$ otherwise. The payoff to the designer is $\pi\left(q_{W}\right)-R$, where $W \in N$ is the identity of the agent winning the contest. In a discriminatory contest, the payoffs to the winning agent and to the designer are $R_{W}-e$ and $\pi\left(q_{W}\right)-R_{W}$, respectively.

We find it convenient to express agents' strategies in terms of quality rather than effort. Thus, agents' strategies are denoted by the functions $\left(q_{i}(\theta)\right)_{i \in N}$, with $q_{i}(\theta) \geq \theta$, where $q_{i}(\theta)$ indicates the choice of quality by agent $i \in N$ when his type is $\theta$. We consider regular strategies. A strategy is regular if it is strictly increasing for all $\theta$ and differentiable whenever $q(\theta)>\theta$. Given the agents' strategies, the efforts exerted by agent $i$ of type $\theta_{i}$ are $e_{i}\left(\theta_{i}\right)=q_{i}\left(\theta_{i}\right)-\theta_{i}{ }^{7}$

\section{Agents' equilibrium strategies in nondiscriminatory contests}

In this section, we provide the agents' strategies in any symmetric equilibrium $q^{*}(\theta)$ when they compete in a nondiscriminatory contest with reward $R$.

To formulate agent $i$ 's maximization problem, for $i \in N$, we let agent $j$ 's strategy be

\footnotetext{
${ }^{7}$ We note that although quality is increasing, the effort need not be increasing in $\theta$. It might be the case that a more able contestant needs to put in less effort to reach the desired quality level. See, for example, the equilibrium in Proposition 2 below.
} 
$q^{*}(\theta)$ for every $j \in N \backslash i$. Then, agent $i$ 's expected profits when he is of type $\theta$ and offers quality $q \geq \theta$ are given by his probability of winning times the reward minus the effort; that is, ${ }^{8}$

$$
\operatorname{Pr}_{\left(\theta_{j}\right)_{j \in N \backslash i}}\left(q \geq \max _{j \in N \backslash i} q^{*}\left(\theta_{j}\right)\right) R-(q-\theta) .
$$

When $q \in\left[q^{*}(0), q^{*}(1)\right]$, and $q \geq \theta$, agent $i$ 's expected profits can be written as

$$
\left(F\left(q^{*-1}(q)\right)\right)^{n-1} R-(q-\theta) .
$$

If the level of quality $q$ that maximizes agent $i$ 's expected profits is interior, that is, $q>\theta$, the following first-order condition (FOC) must hold:

$$
q^{* \prime}\left(q^{*-1}(q)\right)=(n-1) F^{\prime}\left(q^{*-1}(q)\right)\left(F\left(q^{*-1}(q)\right)\right)^{n-2} R,
$$

that is, the optimal $q(\theta)$ satisfies

$$
q^{* \prime}\left(q^{*-1}(q(\theta))\right)=(n-1) F^{\prime}\left(q^{*-1}(q(\theta))\right)\left(F\left(q^{*-1}(q(\theta))\right)\right)^{n-2} R .
$$

In the symmetric equilibrium, $q(\theta)=q^{*}(\theta)$. Therefore, the FOC yields the following differential equation that $q^{*}(\theta)$ must satisfy in an interior solution:

$$
q^{* \prime}(\theta)=(n-1) F^{\prime}(\theta)(F(\theta))^{n-2} R .
$$

The solution of this equation is given by:

$$
q^{*}(\theta)=F(\theta)^{n-1} R+\eta
$$

for some $\eta \in \mathbb{R}$.

Equation (3) describes the equilibrium strategies over the range of $\theta$ 's that lead to an interior solution, where efforts chosen by agents are strictly positive. However, there may be regions of agent types that provide zero effort, $q^{*}(\theta)=\theta$.

If, at equilibrium, the agents choose a positive level of effort for every $\theta>0$, then they play the strategy-tuple $\left(q_{i}^{I}\right)_{i \in N}$ defined by

$$
q_{i}^{I}(\theta)=F(\theta)^{n-1} R \text { for all } \theta \in[0,1], \text { for } i \in N
$$

\footnotetext{
${ }^{8}$ Since $q^{*}(\theta)$ is strictly increasing, we ignore the possibility of ties as they occur with probability zero.
} 
because the constant $\eta$ must be zero in this case (at equilibrium, $\left.q_{i}^{I}(0)=0\right) .{ }^{9}$ Similarly, if the agents choose at equilibrium zero level of effort for every $\theta$, then they follow the strategy profile $\left(q_{i}^{C}\right)_{i \in N}$ defined by

$$
q_{i}^{C}(\theta)=\theta \text { for all } \theta \in[0,1], \text { for } i \in N .
$$

The equilibrium may also involve strategies where the agents choose a positive level of effort for some interval of types whereas they select zero effort for another interval. For example, they could follow the strategy profiles $\left(q_{i}^{C I}\right)_{i \in N}$ or $\left(q_{i}^{I C}\right)_{i \in N}$ defined by

$$
q_{i}^{C I}(\theta)=\left\{\begin{array}{lr}
\theta & \text { for all } \theta \in\left[0, \alpha_{1}\right] \\
F(\theta)^{n-1} R+\eta & \text { for all } \theta \in\left(\alpha_{1}, 1\right]
\end{array}\right.
$$

and

$$
q_{i}^{I C}(\theta)= \begin{cases}F(\theta)^{n-1} R & \text { for all } \theta \in\left[0, \beta_{1}\right) \\ \theta & \text { for all } \theta \in\left[\beta_{1}, 1\right]\end{cases}
$$

for $i \in N$, where $\eta=-F\left(\alpha_{1}\right)^{n-1} R+\alpha_{1}$ in the strategy profile $\left(q_{i}^{C I}\right)_{i \in N}$, and $F\left(\beta_{1}\right)^{n-1} R=$ $\beta_{1}$ in the strategy profile $\left(q_{i}^{I C}\right)_{i \in N}$ because, as we will show, equilibrium strategies are continuous in a symmetric equilibrium. ${ }^{10}$

Before stating the general result for arbitrary distribution functions, we first consider convex and concave distribution functions. Assume first that $F(\theta)$ is convex and let $\alpha_{1}$ denote the value of $\theta$ at which the function $F^{n-1}(\theta) R-\theta$ is minimized, if this function is first decreasing and then increasing. We note that for a convex $F(\theta)$, it is always the case that $F^{\prime}(0) \leq 1 \leq F^{\prime}(1)$. As Proposition 1 shows, the equilibrium strategies assume a simple form, although the form depends on the level of the reward. ${ }^{11}$

\footnotetext{
${ }^{9}$ If all agents $i \in N \backslash j$ choose their quality according to $q_{i}^{I}($.$) , then agent j$ of type $\theta$ obtains the same payoff independent of the quality $q \geq \theta$ he selects, provided $q$ is in the support of the other agents' strategies (see Claim 3 in the proof of Theorem 1). A similar phenomenon occurs when the equilibria of the contests we consider include a range of types that choose a positive level of effort.

${ }^{10}$ Note that the notation we use regarding the boundaries of the intervals follows the convention that any agent type $\theta>0$ who chooses zero effort belongs to the corner equilibrium region. For example, in the $I C$ equilibrium, $F\left(\alpha_{1}\right)^{n-1} R+\eta=\alpha_{1}$ and in the $C I$ equilibrium, $F\left(\beta_{1}\right)^{n-1} R=\beta_{1}$; both $\alpha_{1}$ and $\beta_{1}$ are taken to be in the corner region.

${ }^{11}$ The proofs of the next two proposition are omitted as they are easy corollaries of the general equilibrium characterization provided in Theorem 1.
} 
Proposition 1 Consider a nondiscriminatory contest with a reward $R$. Assume that $F(\theta)$ is convex, that is, $F^{\prime \prime}(\theta) \geq 0$ for all $\theta \in[0,1]$.

(a) If $R \leq \frac{1}{F^{\prime}(1)}$, then an equilibrium is given by $\left(q_{i}^{C}\right)_{i \in N}$.

(b) If $R \in\left(\frac{1}{F^{\prime}(1)}, \frac{1}{F^{\prime}(0)}\right)$, then an equilibrium is given by $\left(q_{i}^{C I}\right)_{i \in N}$.

(c) If $R \geq \frac{1}{F^{\prime}(0)}$, then an equilibrium is given by $\left(q_{i}^{I}\right)_{i \in N}$.

An agent's incentive to exert effort depends on two parameters: the reward $R$ and the increase in the probability of winning due to the effort, which depends on the density $F^{\prime}($.). When the reward $R$ is very small, $R \leq \frac{1}{F^{\prime}(1)}$, then it is never worthwhile to exert any effort and the equilibrium strategies are $\left(q_{i}^{C}\right)_{i \in N}$. When the reward is higher, $R \in\left(\frac{1}{F^{\prime}(1)}, \frac{1}{F^{\prime}(0)}\right)$, then an agent has an incentive to exert effort if the density of $\theta$ (of the rival) is high enough which, given that the function $F(\theta)$ is convex, happens for high values of $\theta$. Thus, the equilibrium involves zero effort for low $\theta$ 's and positive effort for high $\theta$ 's, that is, the strategy profile is $\left(q_{i}^{C I}\right)_{i \in N}$. Similar reasoning explains why the equilibrium profile is $\left(q_{i}^{I}\right)_{i \in N}$ if $R \geq \frac{1}{F^{\prime}(0)}$.

The next simple case is an environment where $F(\theta)$ is concave with two agents denoted by $A$ and $B \cdot{ }^{12}$, In this case, $F^{\prime}(1) \leq 1 \leq F^{\prime}(0)$. Let $\beta_{1}$ denote the value of $\theta>0$ at which the function $F(\theta) R-\theta=0$, in the case such a point exists.

Proposition 2 Consider a nondiscriminatory contest with a reward $R$ and $n=2$. Assume that $F(\theta)$ is concave, that is, $F^{\prime \prime}(\theta) \leq 0$ for all $\theta \in[0,1]$.

(a) If $R \leq \frac{1}{F^{\prime}(0)}$, then an equilibrium is given by $\left(q_{A}^{C}, q_{B}^{C}\right)$.

(b) If $R \in\left(\frac{1}{F^{\prime}(0)}, 1\right)$, then an equilibrium is given by $\left(q_{A}^{I C}, q_{B}^{I C}\right)$.

(c) If $R \geq 1$, then an equilibrium is given by $\left(q_{A}^{I}, q_{B}^{I}\right)$.

The intuition underlying Proposition 2 is similar to that for Proposition 1. The only difference is that the concavity of the function $F(\theta)$ implies that the incentive to exert effort is higher for low values of $\theta$ than for high values of $\theta$. Hence effort might indeed go down in $\theta$, while quality offered is always increasing in $\theta$.

The general case is addressed in Theorem 1, which explicitly describes equilibrium strategies for any differentiable and atomless distribution function $F(\theta)$. Prior to presenting the theorem, we below describe an intuition behind the derivation of the symmetric equilibrium strategies, which are necessarily continuous.

\footnotetext{
${ }^{12}$ Proposition 2 holds for every number of agents, $n$, provided that $F^{n-1}$ is concave.
} 
There are two types of regions in terms of quality $q$. We let Region $I$ be the region of qualities that are reached when agents choose positive levels of effort, that is, $q^{*-1}(q)<q$ and thus the equilibrium is characterized by equation (3). ${ }^{13}$ We let Region $C$ be the one that is reached when agents choose zero effort, that is, $q^{*-1}(q)=q$, or $q^{*}(\theta)=\theta$.

To derive the conditions that an equilibrium must satisfy, we consider the case where agents' strategies lie in Region $I$ for $\theta \in\left(\theta_{1}, \theta_{2}\right)$ and in Region $C$ for $\theta \in\left[\theta_{2}, \theta_{3}\right]$. First, the continuity of the equilibrium strategies implies that $F\left(\theta_{2}\right)^{n-1} R+\eta=\theta_{2}$; hence, $\eta=\theta_{2}-F\left(\theta_{2}\right)^{n-1} R$. Second, for any $\theta \in\left[\theta_{2}, \theta_{3}\right]$, for $q^{*}(\theta)=\theta$ to be the optimal choice, an agent's profits for this choice cannot be lower than his profits for any $q \in\left(\theta, \theta_{3}\right]$, i.e., $F(\theta)^{n-1} R \geq F(q)^{n-1} R-(q-\theta)$, or

$$
F(q)^{n-1} R-q \leq F(\theta)^{n-1} R-\theta \text { for any } \theta \in\left[\theta_{2}, \theta_{3}\right], q \in\left(\theta, \theta_{3}\right] .
$$

Finally, the non-negativity of effort implies that $F(\theta)^{n-1} R+\eta \geq \theta$ for any $\theta$ in $\left(\theta_{1}, \theta_{2}\right)$, or

$$
F\left(\theta_{2}\right)^{n-1} R-\theta_{2} \leq F(\theta)^{n-1} R-\theta \text { for any } \theta \in\left(\theta_{1}, \theta_{2}\right) .
$$

Equation (4) must be satisfied for any interval of agent types whose quality choice belongs to Region $C$, thereby implying that the function $F(\theta)^{n-1} R-\theta$ is non-increasing in such an interval. Equation (5) must be satisfied for any interval of agent types whose quality choice belongs to Region $I$. This equation requires that the value of the function $F(\theta)^{n-1} R-\theta$ at the upper bound of the interval cannot be higher than the value at any other $\theta$ in the interval. These conditions are shown to describe an equilibrium in Theorem 1, where the boundaries of the regions are characterized as well. ${ }^{14}$ The theorem is stated for the case where the function $F(\theta)^{n-1} R-\theta$ is decreasing at $\theta=0$, that is, $(n-1) F(0)^{n-2} F^{\prime}(0) R-1<0$, which always holds when $n>2$. After the theorem, we indicate the small changes necessary to state the results for the other cases with two agents.

Theorem 1 Consider a nondiscriminatory contest with a reward $R$. Assume that $F(\theta)^{n-1} R-$ $\theta$ has a finite set of local minima. ${ }^{15}$ Also, assume that $F(\theta)^{n-1} R-\theta$ is decreasing at $\theta=0$.

\footnotetext{
${ }^{13}$ Region $I$ can be the union of several intervals. If this is the case, the parameter $\eta$ changes from one interval to another.

${ }^{14}$ All proofs are relegated to the appendix.

${ }^{15}$ This is a very mild requirement that is satisfied by any polynomial and any commonly used distribution function.
} 


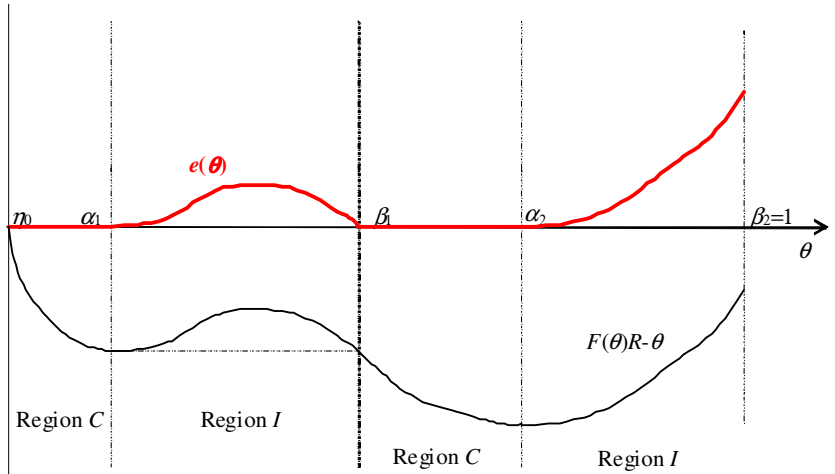

Figure 1: Equilibrium effort in a nondiscriminatory contest for two agents.

We recursively define a finite sequence of critical types $\beta_{0}<\alpha_{1}<\beta_{1}<\alpha_{2}<\ldots$ by $\beta_{0}=0$ and:

(i) given that $\beta_{m-1}<1$ has been defined, $\alpha_{m}$, for $m \geq 1$, is equal to 1 if $F(\theta)^{n-1} R-\theta$ is decreasing in $\left[\beta_{m-1}, 1\right]$; otherwise, $\alpha_{m}$ is the first agent type $\theta$ larger than $\beta_{m-1}$ that is a strict local minimum of $F(\theta)^{n-1} R-\theta$.

(ii) given that $\alpha_{m}<1$ has been defined, $\beta_{m}$, for $m \geq 1$, is equal to 1 if $\alpha_{m}$ is a global minimum; otherwise, $\beta_{m}$ is the first agent type $\theta$ larger than $\alpha_{m}$ for which $F\left(\beta_{m}\right)^{n-1} R-$ $\beta_{m}=F\left(\alpha_{m}\right)^{n-1} R-\alpha_{m}$ that is not a local minimum of the function $F(\theta)^{n-1} R-\theta$.

Then, the unique symmetric equilibrium is:

$$
\begin{aligned}
& \left.q^{*}(\theta)=F(\theta)^{n-1} R+\alpha_{m}-F\left(\alpha_{m}\right)^{n-1} R \text { for } \theta \in\left[\alpha_{m}, \beta_{m}\right], m \geq 1 \text { (Region } I\right) \\
& q^{*}(\theta)=\theta \quad \text { for } \theta \in\left[\beta_{m-1}, \alpha_{m}\right], m \geq 1(\text { Region } C) .
\end{aligned}
$$

Figure 1 represents for the case $n=2$ the equilibrium effort levels $e(\theta)$ and the function $F(\theta) R-\theta$ if $F^{\prime}(0) R-1<0 .{ }^{16} 17$

When $F(\theta)^{n-1} R-\theta$ is increasing at $\theta=0$, Theorem 1 still holds, and the recursive definition of the sequences $\left(\alpha_{m}\right)_{m}$ and $\left(\beta_{m}\right)_{m}$ is similar, except that we start with $\alpha_{1}=0$ and $\beta_{0}$ does not exist. Similarly, if the derivative of $F(\theta)^{n-1} R-\theta$ at $\theta=0$ is zero, the

\footnotetext{
${ }^{16}$ We note that, for $n=2$, the proposed strategies constitute the unique equilibrium in monotonic strategies if the set $\left\{\theta \in[0,1] / F^{\prime}(\theta)=1 / R\right\}$ has zero measure (see Pérez-Castrillo and Wettstein, 2012).

${ }^{17}$ The equilibrium construction bears some similarities to the construction in Seel (2014), where the informed player's bid can also be zero for an interval of types (in his case, the type corresponds to the size of the player's initial advantage).
} 
sequences will be the same as in Theorem 1 unless $F(\theta)^{n-1} R-\theta>0$ for some interval $(0, \widehat{\theta})$ with $\widehat{\theta}>0$, in which case the definition of the sequences starts with $\alpha_{1}=0$.

\section{Agents' equilibrium strategies in discriminatory con- tests}

We now analyze agents' strategies in discriminatory contests when $n=2$. We denote $N=\{A, B\}$ and assume that $R_{A}>R_{B}$. Formulating the agents' maximization problems in the same manner as in the nondiscriminatory case, we obtain the following FOCs for agents $A$ and $B$, when they exert positive efforts:

$$
\begin{aligned}
& q_{B}^{\prime}\left(q_{B}^{-1}(q)\right)=F^{\prime}\left(q_{B}^{-1}(q)\right) R_{A} . \\
& q_{A}^{\prime}\left(q_{A}^{-1}(q)\right)=F^{\prime}\left(q_{A}^{-1}(q)\right) R_{B} .
\end{aligned}
$$

The solution of the system of differential equations is given by

$$
\begin{gathered}
q_{A}(\theta)=F(\theta) R_{B}+\eta_{A}, \\
q_{B}(\theta)=F(\theta) R_{A}+\eta_{B},
\end{gathered}
$$

for some $\eta_{A}, \eta_{B} \in \mathbb{R} .^{18}$

As we found in our analysis of the agents' equilibrium strategies in nondiscriminatory contests, there are also regions where agents put in zero effort. Furthermore, in discriminatory contests, discontinuities in an agent's strategy cannot be ruled out, that is, a quality interval that is never reached by an agent even though he offers qualities below and above that interval may exist.

As we show in the Appendix (Lemmas 1 and 2), only three strategy configurations can emerge in equilibrium. We call Region $I$ the set of quality levels $q$ that are reached when both agents play according to the interior solution; that is, $q \in I$ if $q_{A}^{-1}(q)<q$ and $q_{B}^{-1}(q)<q$. Also, we call Region $C$ the set of qualities reached when both agents put in zero effort; that is, $q \in C$ if $q_{A}^{-1}(q)=q_{B}^{-1}(q)=q$. The remaining region, which

\footnotetext{
${ }^{18}$ When there are $n>2$ agents there exist several possibilities for discrimination, each leading to a different set of conditions and equations. Furthermore, the solution of the resulting systems of differential equations cannot be obtained in a closed form.
} 

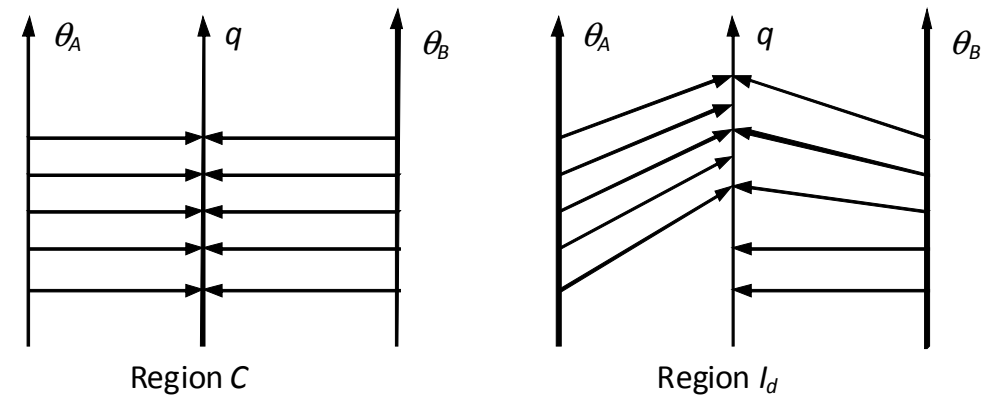

Figure 2: Choice of quality by the two agents in an interval of Region $C$ and in an interval of Region $I_{d}$.

does not exist in nondiscriminatory contests, corresponds to quality levels reached by $B$ through zero effort, but never offered by agent $A$. More precisely, Region $J$ is given by the set of qualities $q$ such that $q_{A}^{-1}(q)$ does not exist and $q_{B}^{-1}(q)=q$. Moreover, Region $J$ always appears before Region $I$. Therefore, the range of qualities in equilibrium can be split into two types of intervals, each of which belong to either Region $C$ or Region $I_{d}$, where Region $I_{d}$ is given by an interval of (lower) qualities that is never reached by agent $A$ and an interval of (higher) qualities that is reached by both agents contributing a positive effort. We note that in discriminatory contests, Region $I_{d}$ plays a similar role to Region $I$ in nondiscriminatory contests. Figure 2 depicts the choice of quality (represented by the middle vertical axis) for both agents as a function of the agent types $\theta_{A}$ and $\theta_{B}$ (represented by the vertical axes on both sides) in two quality intervals, one belonging to region $C$ and another to region $I_{d} \cdot{ }^{19}$

At equilibrium, for any $\theta$, agent $A$ 's effort is greater than or equal to agent $B$ 's effort, that is, $e_{A}(\theta)-e_{B}(\theta) \geq 0$. Efforts are the same in Region $C$ and the inequality is strict for the values of $\theta$ in the lower part of Region $I_{d}$ (we called that Region $J$ previously): agent $B$ provides zero effort whereas agent $A$ chooses a positive effort level. In the upper part of Region $I_{d}, e_{A}(\theta)-e_{B}(\theta)=q_{A}(\theta)-q_{B}(\theta)=\eta_{A}-\eta_{B}-F(\theta)\left(R_{A}-R_{B}\right)$. If we denote by $\bar{\theta}$ the upper bound (in terms of $\theta$ ) of the interval which is in Region $I_{d}$, then at equilibrium $q_{A}(\bar{\theta})=q_{B}(\bar{\theta})$, which implies $\eta_{A}-\eta_{B}=F(\bar{\theta})\left(R_{A}-R_{B}\right)$. Therefore, in

\footnotetext{
${ }^{19}$ The interval in region $I_{d}$ depicted in Figure 2 corresponds to an interval whose upper bounds (in terms of $\theta$ ) are $\theta_{A}=\theta_{B}=1$. If this interval were followed by an interval in region $C$, the transition to the $C$ interval would be continuous, that is the top arrows would be horizontal.
} 
this part of Region $I_{d}, e_{A}(\theta)-e_{B}(\theta)=(F(\bar{\theta})-F(\theta))\left(R_{A}-R_{B}\right)>0$ for all $\theta<\bar{\theta}$. The distance in effort decreases as $\theta$ increases and it vanishes at $\theta=\bar{\theta}$.

So far we have highlighted several properties of equilibrium strategies in discriminatory contests for general distribution functions. While the characterization of equilibrium is cumbersome in a general environment, simple characterization is possible in a fairly permissive class of distributions.

First, we discuss the possibility of a corner solution. It is intuitive that such a solution emerges if the prizes allocated in the contest are very small. Proposition 3 goes a step forward and provides a necessary and sufficient condition for Region $C$ to constitute the only equilibrium in the contest. In such an equilibrium, both agents choose zero effort for every $\theta \in[0,1]$, a strategy profile that we have denoted by $\left(q_{A}^{C}, q_{B}^{C}\right)$.

Proposition 3 The strategy profile $\left(q_{A}^{C}, q_{B}^{C}\right)$ constitutes an equilibrium of the contest $\left(R_{A}, R_{B}\right)$ if and only if the function $F(\theta) R_{A}-\theta$ is non-increasing in $\theta$ for all $\theta \in[0,1]$. Moreover, if the set $\left\{\theta \in[0,1] \mid F^{\prime}(\theta)=1 / R_{A}\right\}$ has zero measure, then no other equilibrium exists.

Second, if the function $F(\theta) R_{A}-\theta$ is non-decreasing for all $\theta \in[0,1]$ (which implies $R_{A}$ is high enough), then, in equilibrium, the qualities offered always lie in Region $I_{d}$. That is, agents follow the strategy profile $\left(q_{A}^{I_{d}}, q_{B}^{I_{d}}\right)$, defined by

$$
\begin{array}{lr}
q_{A}^{I_{d}}(\theta)=F(\theta) R_{B}+\gamma & \text { for all } \theta \in[0,1] \\
q_{B}^{I_{d}}(\theta)=\left\{\begin{array}{lr}
\theta & \text { for all } \theta \in[0, \gamma] \\
F(\theta) R_{A}+\gamma-F(\gamma) R_{A} & \text { for all } \theta \in(\gamma, 1]
\end{array}\right.
\end{array}
$$

where $\gamma$ solves $R_{B}=[1-F(\gamma)] R_{A}$; hence, $\gamma=F^{-1}\left(1-\frac{R_{B}}{R_{A}}\right) \cdot{ }^{20} 21$

The whole class of distribution functions and rewards for which the strategy profile $\left(q_{A}^{I_{d}}, q_{B}^{I_{d}}\right)$ is an equilibrium is provided in Proposition 4.

${ }^{20}$ The qualities chosen by the agents as well as the cut-off $\gamma$ in the profile $\left(q_{A}^{I_{d}}, q_{B}^{I_{d}}\right)$ depend on the particular function $F(\theta)$ and the rewards $R_{A}$ and $R_{B}$. We do not express this dependence on $\left(q_{A}^{I_{d}}, q_{B}^{I_{d}}\right)$ to keep the notation simple.

${ }^{21}$ The critical type $\gamma$ varies continuously in the "size" of discrimination, as measured by the ratio $\frac{R_{B}}{R_{A}}$. Also, when $R_{A}=R_{B}=R$, then $\gamma=0$, in which case the strategy profile $\left(q_{A}^{I_{d}}, q_{B}^{I_{d}}\right)$ coincides with the strategy profile $\left(q_{A}^{I}, q_{B}^{I}\right)$, defined for the nondiscriminatory contest with reward $R$. 
Proposition 4 The strategy profile $\left(q_{A}^{I_{d}}, q_{B}^{I_{d}}\right)$ constitutes an equilibrium of the contest $\left(R_{A}, R_{B}\right)$ if and only if the following two conditions hold:

$$
\begin{aligned}
& F(\theta) R_{A}-\theta \leq F(\gamma) R_{A}-\gamma \text { for all } \theta \leq \gamma \\
& F(\theta) R_{A}-\theta \geq F(\gamma) R_{A}-\gamma \text { for all } \theta \geq \gamma
\end{aligned}
$$

In particular, $\left(q_{A}^{I_{d}}, q_{B}^{I_{d}}\right)$ is an equilibrium if $F(\theta) R_{A}-\theta$ is non-decreasing in $\theta$ for all $\theta \in[0,1]$. Furthermore, the equilibrium is unique if inequalities (10) and (11) are strict for all $\theta \neq \gamma$.

The structure of the $\left(q_{A}^{I_{d}}, q_{B}^{I_{d}}\right)$ equilibrium can be explained as follows. Since $R_{A}$ is high enough, agent $A$ has an incentive to always exert positive effort. The lower reward of agent $B$ makes him less aggressive than $A$. He puts in zero effort if his type is very low. This, in turn, provides an incentive for agent $A$ to jump to a positive effort $\gamma>0$ when $\theta_{A}=0$ and earn strictly positive profits, whereas profits would be zero with $q_{A}(0)=0$.

When the function $F(\theta) R_{A}-\theta$ is increasing in some intervals of $\theta$ 's and decreasing in others, then the equilibrium will include intervals of quality that lie in Region $I_{d}$ and, often, others that lie in Region $C$. Next, we consider two families of distribution functions where this happens, namely convex or concave distribution functions.

Theorem 2 characterizes the structure of equilibrium strategies when the function $F(\theta)$ is convex. It extends Proposition 1 to discriminatory contests. As in Proposition 1, the equilibrium configuration can start either with a corner region $C$ or an interior region, $I_{d}$ in discriminatory contests. Given the convexity of $F(\theta)$, if the equilibrium starts with an $I_{d}$ region, it coincides with an $I_{d}$ equilibrium throughout. On the other hand, if the equilibrium starts with a $C$ region then, as we did in Theorem 1 , we can define $\beta_{0}=0$ and look for $\alpha_{1}$. If the function $F(\theta) R_{A}-\theta$ is always decreasing, then $\alpha_{1}=1$ and the equilibrium is given by $\left(q_{A}^{C}, q_{B}^{C}\right)$. Otherwise, the equilibrium consists of a $C$ and then an $I_{d}$ region. Instead of looking for one critical value, we need to find both $\alpha_{1}$ and $\gamma_{1}$, where $\alpha_{1}$ determines the end of the corner region and $\gamma_{1}$ determines the size of the jump in the $I_{d}$ region. Again, by convexity, once the equilibrium enters an $I_{d}$ region, it stays in it. 
To simplify the presentation of the theorem, we define the strategy profile $\left(q_{A}^{C I_{d}}, q_{B}^{C I_{d}}\right)$ :

$$
\begin{aligned}
& q_{A}^{C I_{d}}(\theta)=\left\{\begin{array}{lr}
\theta & \text { for all } \theta \in[0, \alpha] \\
F(\theta) R_{B}+\gamma-F(\alpha) R_{B} & \text { for all } \theta \in(\alpha, 1]
\end{array}\right. \\
& q_{B}^{C I_{d}}(\theta)= \begin{cases}\theta & \text { for all } \theta \in[0, \gamma] \\
F(\theta) R_{A}+\gamma-F(\gamma) R_{A} & \text { for all } \theta \in(\gamma, 1]\end{cases}
\end{aligned}
$$

where $\alpha$ and $\gamma$ solve

$$
\begin{gathered}
{[1-F(\alpha)] R_{B}=[1-F(\gamma)] R_{A}} \\
F(\alpha) R_{A}-\alpha=F(\gamma) R_{A}-\gamma
\end{gathered}
$$

Under the strategy profile $\left(q_{A}^{C I_{d}}, q_{B}^{C I_{d}}\right)$, both agents exert zero effort up to a threshold value of $\theta$ and choose positive effort levels for higher values. Agent $A$ 's threshold is lower than agent $B$ 's, $(\alpha<\gamma)$ due to the higher reward he obtains if he wins the contest. Finally, while agent $B$ 's strategy is continuous in the type $\theta$, agent $A$ 's strategy entails a discrete jump from $\alpha$ to $\gamma$ at his threshold.

Theorem 2 Let $F(\theta)$ be convex, that is, $F^{\prime \prime}(\theta) \geq 0$ for all $\theta \in[0,1]{ }^{22}$

(a) If $R_{A} \leq \frac{1}{F^{\prime}(1)}$, then an equilibrium is given by $\left(q_{A}^{C}, q_{B}^{C}\right)$.

(b) If either $R_{A} \in\left(\frac{1}{F^{\prime}(1)}, 1\right)$ or both $R_{A} \in\left[1, \frac{1}{F^{\prime}(0)}\right)$ and $F\left(R_{A}-R_{B}\right) R_{A}-\left(R_{A}-R_{B}\right) \leq$ 0 , then an equilibrium is given by $\left(q_{A}^{C I_{d}}, q_{B}^{C I_{d}}\right) .{ }^{23}$

(c) If either $R_{A} \geq \frac{1}{F^{\prime}(0)}$ or both $R_{A} \in\left[1, \frac{1}{F^{\prime}(0)}\right)$ and $F\left(R_{A}-R_{B}\right) R_{A}-\left(R_{A}-R_{B}\right)>0$, then an equilibrium is given by $\left(q_{A}^{I_{d}}, q_{B}^{I_{d}}\right)$.

Furthermore, these are the unique equilibrium configurations.

We point out that when $R_{A} \in\left[1, \frac{1}{F^{\prime}(0)}\right)$, the condition $F\left(R_{A}-R_{B}\right) R_{A}-\left(R_{A}-R_{B}\right)>$ 0 is equivalent to $R_{A}-R_{B}>p\left(R_{A}\right)$, where we denote by $p\left(R_{A}\right)$ the unique strictly positive $p$ that satisfies $F(p) R_{A}-p=0$. Thus, when $R_{A} \in\left[1, \frac{1}{F^{\prime}(0)}\right)$, we are in case (c) if $R_{B}$ is not "close" to $R_{A}$.

\footnotetext{
${ }^{22}$ The theorem holds for all weakly convex functions provided that the minimum of $F(\theta) R_{A}-\theta$, if it exists, is unique.

${ }^{23}$ The critical values $\gamma$ and $\alpha$ are uniquely defined and continuous in $R_{A}$ and $R_{B}$ in this region. If $R_{A}=R_{B}$, the system (12)-(13) does not have a unique solution. The limits of $\gamma$ and $\alpha$ when $R_{B}$ converges to $R_{A}=R$ correspond to the solution of (12)-(13) that satisfies $\gamma=\alpha$ and $F^{\prime}(\alpha) R=1$, which yields the strategy profile $\left(q_{A}^{C I}, q_{B}^{C I}\right)$.
} 


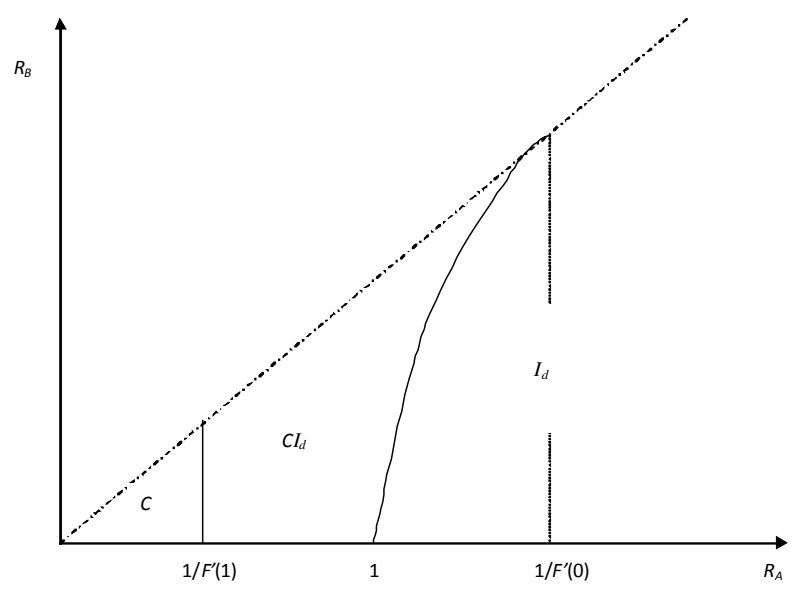

Figure 3: Equilibrium configuration when $F($.$) is convex.$

Figure 3 depicts the equilibrium configuration as a function of the prizes $R_{A}$ and $R_{B}$ for the class of convex distribution functions. The implicit equation yielding the border between the regions $C I_{d}$ and $I_{d}$ is given by $F\left(R_{A}-R_{B}\right) R_{A}-\left(R_{A}-R_{B}\right)=0$. It describes $R_{B}$ as a function of $R_{A}$ starting from $R_{B}=0$ when $R_{A}=1$. The slope of the function is larger than 1 and it converges to 1 when $R_{A}$ approaches $\frac{1}{F^{\prime}(0)} \cdot{ }^{24}$

When $R_{A}$ is small enough, the whole equilibrium is in Region $C$, and when $R_{A}$ is large enough, the equilibrium is in Region $I_{d}$ where agent $A$ puts in a positive effort at $\theta=0$ and agent $B$ starts to exert a positive effort only for large enough $\theta$ 's. The same occurs when $R_{A}$ is intermediate but substantially larger than $R_{B}$. The intuition for these two cases is similar to that provided after Propositions 3 and 4, namely contestants do not have an incentive to exert any effort if the reward is low whereas competition in efforts arises if the reward is high.

When $R_{A}$ is intermediate and, depending on $R_{A}$, not too much higher than $R_{B}$, the equilibrium entails a corner solution for low values of $\theta$ and an interior solution for high values (the Region $C I_{d}$ ). The reason for a positive effort being exerted at higher values of $\theta$ can be traced back to the larger density at higher values of $\theta$ due to the convexity of the distribution function $F(\theta)$. If the density is high, increasing the effort implies a large

\footnotetext{
${ }^{24}$ Taking the total differential of $F\left(R_{A}-R_{B}\right) R_{A}-\left(R_{A}-R_{B}\right)=0$ we obtain $\frac{d R_{B}}{d R_{A}}=$ $\frac{F^{\prime}\left(R_{A}-R_{B}\right) R_{A}+F\left(R_{A}-R_{B}\right)-1}{F^{\prime}\left(R_{A}-R_{B}\right) R_{A}-1}=1+\frac{F\left(R_{A}-R_{B}\right)}{\frac{F^{\prime}\left(R_{A}-R_{B}\right)\left(R_{A}-R_{B}\right)}{F\left(R_{A}-R_{B}\right)}-1}$. The denominator is positive due to the convexity of the distribution function $F\left(\right.$.), hence the slope exceeds 1 (and equals 1 when $R_{A}=R_{B}$ ).
} 
increase in the probability of winning the contest hence, a larger payoff.

As we have mentioned above, agent $A$ 's strategy is discontinuous at $\theta=\alpha$, where he jumps from zero effort to $e=\gamma-\alpha>0$. The intuition for the discontinuity is the following. Agent $B$ exerts zero effort for $\theta \in[0, \gamma]$. This implies, because of the convexity of the distribution function of types, that agent $A$ 's marginal benefits, for a given $\theta$, are increasing in $q_{A}$. The marginal benefits are negative for low values of $q_{A}$ including $q_{A}=\alpha$. However, marginal profits start being positive before $q_{A}=\gamma$ and profits are the same for $q_{A}=\alpha$ and $q_{A}=\gamma$. The reason that agent $A$ does not increase effort further is that agent $B$ also selects positive effort from $\theta=\gamma$ onwards, which reduces agent $A$ 's incentive to exert a higher effort.

Figure 4 illustrates the quality choices by the agents in an example where $F(\theta)=\theta^{3}$, $R_{A}=1$ and $R_{B}=0.666$, which implies that the solution lies in region (b) in Theorem 2. Both agents choose zero effort as long as $\theta<\alpha=0.416$ at which point agent $A$ jumps to $q_{A}=\gamma=0.725$ and chooses a positive effort thereafter. On the other hand, agent $B$ continues with zero effort up to $\theta=0.725$ and chooses a positive effort for higher $\theta$ 's.

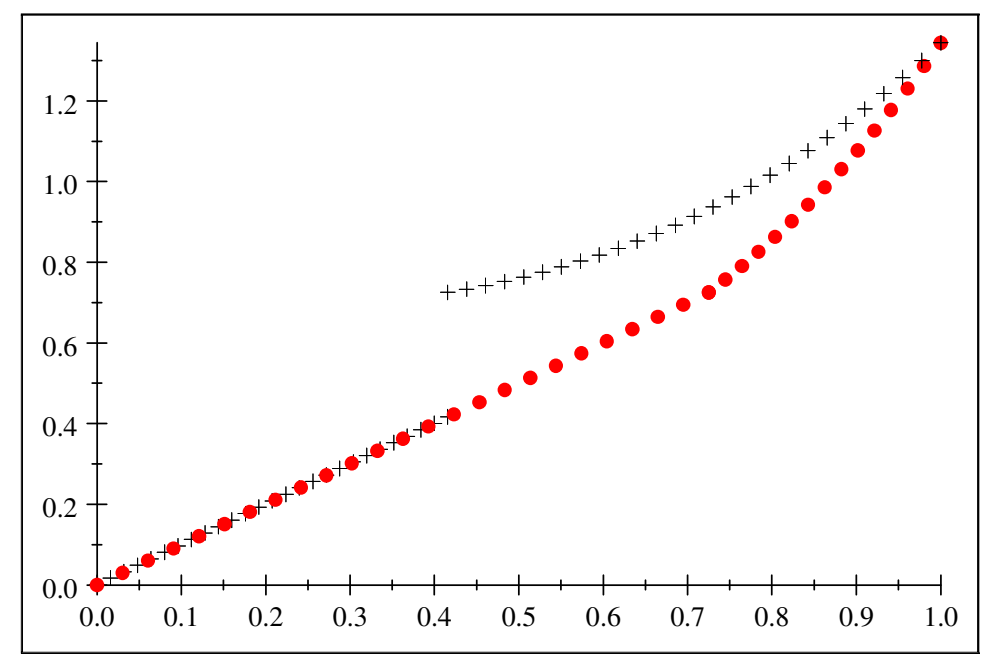

Figure 4: Quality choices $q_{A}$ (black crosses) and $q_{B}$ (red dots) if $F(\theta)=\theta^{3}, R_{A}=1$ and $R_{B}=0,666$.

We now characterize the equilibrium strategies when the function $F(\theta)$ is concave. Theorem 3 generalizes Proposition 2 for discriminatory contests. The intuition underlying the equilibrium and the transition between the regions is similar to the one appearing before Theorem 2, with the natural modifications due to the concavity of $F(\theta)$. 
We define the strategy profile $\left(q_{A}^{I_{d} C}, q_{B}^{I_{d} C}\right)$ as follows:

$$
\begin{aligned}
& q_{A}^{I_{d} C}(\theta)=\left\{\begin{array}{lr}
F(\theta) R_{B}+\gamma & \text { for all } \theta \in[0, \beta) \\
\theta & \text { for all } \theta \in[\beta, 1]
\end{array}\right. \\
& q_{B}^{I_{d} C}(\theta)=\left\{\begin{array}{lr}
\theta & \text { for all } \theta \in[0, \gamma] \cup[\beta, 1] \\
F(\theta) R_{A}+\gamma-F(\gamma) R_{A} \text { for all } \theta \in(\gamma, \beta)
\end{array}\right.
\end{aligned}
$$

where $\gamma$ and $\beta$ solve

$$
\begin{aligned}
\gamma & =\beta-F(\beta) R_{B} \\
F(\gamma) R_{A}-\gamma & =F(\beta) R_{A}-\beta .
\end{aligned}
$$

When agents follow the strategy profile $\left(q_{A}^{I_{d} C}, q_{B}^{I_{d} C}\right)$, they exert zero effort above the same threshold value of $\theta$. For low values of $\theta$, they follow an interior solution where agent $A$ has an incentive to exert a strictly positive effort even when $\theta_{A}=0$, whereas agent $B$ only exerts a positive effort above a certain threshold of $\theta_{B}$.

Theorem 3 Let $F(\theta)$ be concave, that is, $F^{\prime \prime}(\theta) \leq 0$ for all $\theta \in[0,1]{ }^{25}$

(a) If $R_{A} \leq \frac{1}{F^{\prime}(0)}$, then an equilibrium is given by $\left(q_{A}^{C}, q_{B}^{C}\right)$.

(b) If either $R_{A} \in\left(\frac{1}{F^{\prime}(0)}, 1\right)$ or both $R_{A} \in\left[1, \frac{1}{F^{\prime}(1)}\right)$ and $F\left(1-R_{B}\right) R_{A}-\left(1-R_{B}\right)>R_{A}-1$, then an equilibrium is given by $\left(q_{A}^{I_{d} C}, q_{B}^{I_{d} C}\right) \cdot{ }^{26}$

(c) If either $R_{A} \geq \frac{1}{F^{\prime}(1)}$ or both $R_{A} \in\left[1, \frac{1}{F^{\prime}(1)}\right)$ and $F\left(1-R_{B}\right) R_{A}-\left(1-R_{B}\right) \leq R_{A}-1$, then an equilibrium is given by $\left(q_{A}^{I_{d}}, q_{B}^{I_{d}}\right)$.

Furthermore, these are the unique equilibrium configurations.

Note that when $R_{A} \in\left[1, \frac{1}{F^{\prime}(1)}\right)$, the condition $F\left(1-R_{B}\right) R_{A}-\left(1-R_{B}\right)>R_{A}-1$ is equivalent to $z\left(R_{A}\right)<1-R_{B}$, where we denote by $z\left(R_{A}\right)$ the unique strictly positive $z$ that satisfies $F(z) R_{A}-z=R_{A}-1$. Thus, when $R_{A} \in\left[1, \frac{1}{F^{\prime}(0)}\right)$, we are in case (b) if $R_{B}$ is "small enough" or not too close to $R_{A}$.

\footnotetext{
${ }^{25}$ The theorem holds for all weakly concave functions provided that the maximum of $F(\theta) R_{A}-\theta$, if it exists, is unique.

${ }^{26}$ The critical values $\beta$ and $\gamma$ are uniquely defined and continuous in $R_{A}$ and $R_{B}$ in this region. If $R_{A}=R_{B}$, the system (14)-(15) does not have a unique solution. The limits of $\beta$ and $\gamma$ when $R_{B}$ converges to $R_{A}=R$ correspond to the solution of (14)-(15) that satisfies $\gamma=0$ and $\beta$ given by the unique positive value for which $F(\beta) R-\beta=0$, which yields the strategy profile $\left(q_{A}^{I C}, q_{B}^{I C}\right)$.
} 
When rewards are quite low or quite high, the agents' behavior is similar to that for the convex case. For intermediate values of reward, they play according to the interior strategy profile for low levels of $\theta$ and exert zero effort for high values of $\theta$. Notice that the corner strategy profile emerges now for high values of $\theta$ because of the low density of $\theta$ due to the concavity of the distribution function.

\section{Designer's payoff and discrimination}

In this section, we address the desirability of discrimination when $n=2$. The designer's benefit $\pi(q)$ depends on the best quality obtained in the contest. We can determine the expected designer's payoff as a function of the agents' strategies, which allows us to discuss the change in the designer's payoff due to discrimination separately for the four possible equilibrium strategy profiles that we identified in Section 4.

The discussion of one of the profiles is trivial. In any contest for which the agents' equilibrium strategy profile is $\left(q_{A}^{C}, q_{B}^{C}\right)$, the designer's revenue is the same, whereas the cost increases with the rewards. Therefore, any contest with positive rewards is dominated by the nondiscriminatory contest with $R=0$ in the sense that the designer's profits are higher if $R=0$. This implies that discrimination can only decrease the designer's payoff. Proposition 5 addresses the other three cases.

Proposition 5 Assume that $\pi^{\prime}(q)>0$ and consider a nondiscriminatory contest $R_{A}=$ $R_{B}=R$ for which an equilibrium is given by the strategy profile $\left(q_{A}, q_{B}\right)$. Consider a marginal change in $\left(R_{A}, R_{B}\right)$ that increases $R_{A}$ by $\varepsilon>0$ and decreases $R_{B}$ by $\varepsilon$.

(a) If $\left(q_{A}, q_{B}\right)=\left(q_{A}^{I}, q_{B}^{I}\right)$ or $\left(q_{A}, q_{B}\right)=\left(q_{A}^{I C}, q_{B}^{I C}\right)$, then the contest is dominated by a discriminatory contest if $R<\frac{2}{F^{\prime}(0)}$.

(b) If $\left(q_{A}, q_{B}\right)=\left(q_{A}^{C I}, q_{B}^{C I}\right)$, then the contest is dominated by a discriminatory contest.

Discrimination in the cases addressed in Proposition 5 is desirable because it elicits higher efforts on the part of the agents. A marginal change in the rewards that leads to a discriminatory contest increases the designer's expected cost. However, this increase in cost is a second-order effect because the infinitesimal change in the rewards is multiplied by infinitesimal changes in the probability of $A$ winning (which go up) and $B$ winning (which go down). On the other hand, the marginal change in the rewards has a first-order 
effect (which, in principle, can be positive or negative) on the expected quality of the innovation, hence, on the designer's expected revenue.

Consider a shift from a nondiscriminatory contest $R$ to a discriminatory contest $\left(R_{A}=\right.$ $\left.R+\varepsilon, R_{B}=R-\varepsilon\right)$ when the initial equilibrium is $\left(q_{A}^{I}, q_{B}^{I}\right)$, which requires that $F^{\prime}(0) R \geq 1$. The increase in $R_{A}$ (and the decrease in $R_{B}$ ) makes player $A$ more aggressive: it gives him an incentive to provide a strictly positive level of quality $(\gamma)$ even if his type were the lowest. Player $B$ becomes less aggressive and prefers not to provide any effort if his type is low (lower than $\gamma$ ). While the initial increase in the effort by player $A$ is $\gamma$ for all the low types, the initial loss of effort by player $B$ depends on the effort that he was exerting in the equilibrium $\left(q_{A}^{I}, q_{B}^{I}\right)$, which is given by the function $F(\theta) R-\theta$. If the initial effort was very low, i.e., if $F^{\prime}(0) R$ is close to 1 , then the increase in $q_{A}$ is larger than the decrease in $q_{B}$. Moreover, this change in qualities offered by low levels of $\theta$ moves up the equilibrium qualities for the whole interval $[0,1]$. This leads to the condition that discrimination is optimal if $F^{\prime}(0) R<2$ whereas it is not otherwise. Moreover, the same intuition explains the condition when the equilibrium in the nondiscriminatory contest is $\left(q_{A}^{I C}, q_{B}^{I C}\right)$, because the region for low values of types under discrimination is also an $I_{d}$ region.

If we look at the changes induced by discrimination when agents' equilibrium behavior under a nondiscriminatory contest is $\left(q_{A}^{C I}, q_{B}^{C I}\right)$ (case $\left.(b)\right)$, the positive effect that we have identified previously also exists. In this case, discrimination induces a discontinuity in agent $A$ 's strategy who now jumps from $q=\alpha$ to $q=\gamma$ when $\theta=\alpha \cdot{ }^{27}$ Moreover, a new effect appears: the stronger incentives for agent $A$ to provide a higher effort translate to a decrease in the cut-off value $\alpha$ from which on he provides a positive effort. Therefore, the corner region $C$ shrinks and the interior region $I$ (which becomes $I_{d}$ ) expands. This additional positive effect makes discrimination always beneficial.

While Proposition 5 shows when it is worthwhile to discriminate for a given strategy profile, it fails to provide actual conditions under which a discriminatory contest dominates a nondiscriminatory one. To show that such an equilibrium profile arises in an optimal nondiscriminatory contest, we first discuss the optimality of discrimination when the

\footnotetext{
${ }^{27}$ When the equilibrium moves from $\left(q_{A}^{I}, q_{B}^{I}\right)$ to $\left(q_{A}^{I_{d}}, q_{B}^{I_{d}}\right)$ or from $\left(q_{A}^{I C}, q_{B}^{I C}\right)$ to $\left(q_{A}^{I_{d} C}, q_{B}^{I_{d} C}\right)$ the jump in agent $A$ 's strategy is similar. However, his strategy is continuous in these cases because the jump happens at $\theta=0$.
} 
designer has a budget constraint. Second, we present an illustrative example and, finally, we provide sufficient conditions in the case of a convex distribution function.

Consider the following environment. ${ }^{28}$ Assume the distribution function is convex, in which case Figure 3 depicts the equilibrium configuration for different reward-pairs. Assume further that the designer has a budget constraint, meaning that the reward cannot be too high; in particular, the upper-bound for the reward is strictly smaller than $1 / F^{\prime}(0)$. If rewards are identical, $R_{A}=R_{B}$, then the equilibrium is (see Proposition 1) either (1) $\left(q_{A}^{C}, q_{B}^{C}\right)$, which involves zero effort, or $(2)\left(q_{A}^{C I}, q_{B}^{C I}\right)$, where effort is positive with positive probability. In case (1), the efforts do not depend on the reward and the optimal contest involves $R=0$. In case (2), perturbing the rewards to $R_{A}+\varepsilon$ and $R_{B}-\varepsilon, \varepsilon>0$, respectively, leads to the equilibrium structure $\left(q_{A}^{C I_{d}}, q_{B}^{C I_{d}}\right)$. Proposition 5 , part (b), tells us that the discriminatory contest dominates the nondiscriminatory contest. Thus, when $R_{A}$ is bounded above by $1 / F^{\prime}(0)$, the optimal contest either involves $R_{A}=R_{B}=0$, or it is discriminatory. The same conclusion can be reached if the budget constraint is between $1 / F^{\prime}(0)$ and $2 / F^{\prime}(0)$ (by part (a) of Proposition 5). This logic also implies that if the distribution function is convex, there is no budget constraint, and a nondiscriminatory contest is optimal, then it must involve either a zero reward or a large reward, in excess of $2 / F^{\prime}(0)$.

We now propose a particular example where the distribution function is uniform.

Example 1. Consider the case where the designer wants to achieve a minimum level of quality $\underline{q}$. That is, any lower quality innovation yields zero revenues for the designer whereas innovations of quality $\underline{q}$ or higher yield a revenue of $\Gamma>0: \pi(q)=0$ for $q \in[0, \underline{q})$ and $\pi(q)=\Gamma$ for $q \geq \underline{q}$. We analyze the case where $\underline{q} \leq 1$. Furthermore, we assume that an agent's type is uniformly distributed, that is, $F(\theta)=\theta$.

In a nondiscriminatory contest $R$, the equilibrium is $\left(q_{A}^{C}, q_{B}^{C}\right)$ if $R \leq 1$ and $\left(q_{A}^{I}, q_{B}^{I}\right)$ if $R>1$. We note that although $\pi(q)$ is not strictly increasing, the result in Proposition 5 (a) still holds because $\pi(1)>\pi(\varepsilon)$ for some $\varepsilon>0 .{ }^{29}$ Therefore, the designer's payoff increases under discrimination if the optimal nondiscriminatory contest implies a reward $R^{*} \in(1,2)$.

\footnotetext{
${ }^{28}$ We thank an anonymous reviewer for suggesting that we analyze this environment.

${ }^{29}$ Proposition 5 (a) holds if $\int_{0}^{R} \pi(q) d q-\pi(R) R<0$, which is the case if $\pi^{\prime}(q)>0$ but it is also the case if the weaker conditions that $\pi(q)$ is nondecreasing and $\pi(1)>\pi(\varepsilon)$ hold.
} 
For $R>1$, easy calculations show that the designer's payoff is $U(R)=\Gamma-\frac{q^{2}}{R^{2}} \Gamma-R$. The optimal $R^{*}$ (if it is higher than 1 ) is $R^{*}=2^{\frac{1}{3}}\left(\underline{q}^{2} \Gamma\right)^{\frac{1}{3}}$. Therefore, discrimination dominates nondiscrimination if two conditions are met: $U\left(2^{\frac{1}{3}}\left(\underline{q}^{2} \Gamma\right)^{\frac{1}{3}}\right)>U(0)=\left(1-\underline{q}^{2}\right) \Gamma$ (which implies that $R^{*}>1$ ) and $2^{\frac{1}{3}}\left(\underline{q}^{2} \Gamma\right)^{\frac{1}{3}}<2$, that is, $\underline{q}^{2} \Gamma \in\left(\frac{3^{\frac{3}{2}}}{2}, 4\right)$, which is satisfied, for example, if $\underline{q}=1$ and $\Gamma=3$.

For illustration, we can compute the optimal discriminatory contest in this numerical example, which is $R_{A}=1.8393$ and $R_{B}=1.6639$. The discriminatory contest yields a payoff of $U(1.8393,1.6639)=0.28228$, improving the payoff obtained in the optimal nondiscriminatory contest, which is $U\left(6^{\frac{1}{3}}\right)=0.27432$.

A more general class of environments where discrimination increases the designer's payoff is given by the following proposition.

Proposition 6 If $F(\theta)$ is a convex function with $F^{\prime}(0)=0$, and $\pi(q)=v i(q)$, with $v>0$ and $i(q)$ a bounded and strictly increasing function of $q$, then discrimination increases the designer's payoff if $v$ is large enough.

Proposition 6 shows that when the distribution of types puts more weight on the higher types, then the designer has an incentive to discriminate if the innovation is very profitable.

\section{Conclusion}

We provided a new setting of contests with asymmetric information where innate abilities and effort combine to generate services or commodities of various qualities. Both the ability and effort of an agent are his private information. The designer, whose revenue depends on the quality of the bid, specifies a contest where the highest quality product is rewarded. We first analyzed strategic behavior in a nondiscriminatory contest, where the reward does not depend on the identity of the winner. We allowed for arbitrary distribution functions and determined the structure of equilibrium strategies and outcomes. The equilibrium agents' strategies consisted of two types of quality intervals: regions where all agents put in a positive effort and those where all agents put in zero effort.

We then analyzed strategic behavior in discriminatory contests where rewards depend on the identity of the winner. Here equilibrium strategies were more complex. We 
provided a qualitative analysis of the structure of the equilibrium strategies for general distribution functions and a full characterization of equilibrium behavior for several classes of functions, in particular if the distribution function is either convex or concave.

We then used the equilibrium analysis to evaluate the designer's payoff. This generated conditions under which the designer prefers a discriminatory to a nondiscriminatory contest. To show that the conditions are not vacuous, we provided parameterized classes of environments where discrimination is beneficial. Our result that discrimination is desirable in a symmetric setting goes against the intuition that when agents are asymmetric, some restrictions imposed on the stronger contestant may increase the designer's payoff.

Our model can handle many familiar scenarios. In addition to innovation, it can be used, for example, to analyze, lobbying activity, procurement settings, promotion competitions and even the design of sporting events. It can also be used to study contest design in the presence of asymmetric contestants and shed further light on the imposition of handicaps or favoritism. Consider for example the environment studied by $\mathrm{Fu}$ (2006). He shows that a college can maximize the expected quality of its students by using handicaps in the admission procedure based on applicants' valuation of education. We do not study handicaps and focus on discrimination via rewards. Our results suggest that even if two candidates are ex-ante identical in every respect, it may still be optimal to benefit one of them in terms of the reward, for example, by decreasing his tuition fee.

The advantages of discrimination extend beyond the framework analyzed in our paper. In particular, they do not rely on the hypothesis that the designer only cares about the best quality offered in the contest. Consider an organization that benefits from the inputs provided by all the contestants. For example, in a promotion contest, the efforts of all the contestants benefit the firm; in a sport or a talent contest, the designer (the spectators) benefits from the performance of all the participants; in a sales contest, the firm benefits from the outcomes realized by all the sales people. Then, Propositions 5 and 6 (with the appropriate modifications) easily extend to this environment.

The model can be extended in several dimensions. A dynamic version would consider two-stage contests where the winners of the first round are paired against each other in the second round. The designer's objective function may also be expanded to include explicit dependence on the agent's innate ability. Moreover, since the analysis was carried out only from the point of view of a single designer, a challenging task for further research 
would be to consider environments with several competing designers.

\section{Appendix}

Proof of Theorem 1. $q^{*}(\theta)$ is differentiable whenever $q^{*}(\theta)>\theta$ by construction. We now show through a series of claims that $q^{*}(\theta)$ is indeed a symmetric equilibrium strategy. Claim 1. The equilibrium quality is well defined, that is, $q^{*}(\theta) \geq \theta$ for any $\theta \in\left[\alpha_{m}, \beta_{m}\right]$ for any $m$.

By construction, $\alpha_{m}$ is a local minimum of the function $F(\theta)^{n-1} R-\theta . \quad \beta_{m}$ is the first instance for which $F(\theta)^{n-1} R-\theta$ also reaches this minimum for $\theta>\alpha_{m}$ and, if this minimum is never reached again, $\beta_{m}=1$. Hence, $q^{*}(\theta)=F(\theta)^{n-1} R-\left(F\left(\alpha_{m}\right)^{n-1} R-\right.$ $\left.\alpha_{m}\right) \geq \theta$ for any $\theta \in\left[\alpha_{m}, \beta_{m}\right]$.

Claim 2. The equilibrium quality $q^{*}(\theta)$ is continuous. It is also strictly increasing in $\theta$ and hence $q^{*}(\theta)$ is a regular strategy.

By definition, $q^{*}(\theta)=\theta$ over all intervals in $C$, and $q^{*}(\theta)=F(\theta)^{n-1} R+\eta$ over all intervals in $I$ (where $\eta$ is different for different intervals). Hence, $q^{*}(\theta)$ is continuous over any interval. Moreover, for any interval $\left[\alpha_{m}, \beta_{m}\right]$ in $I, q^{*}\left(\alpha_{m}\right)=F\left(\alpha_{m}\right)^{n-1} R+\alpha_{m}-$ $F\left(\alpha_{m}\right)^{n-1} R=\alpha_{m}$ and $q^{*}\left(\beta_{m}\right)=F\left(\beta_{m}\right)^{n-1} R+\alpha_{m}-F\left(\alpha_{m}\right)^{n-1} R=\beta_{m}$. Therefore, $q^{*}(\theta)$ is continuous everywhere. Finally, given that $F(\theta)$ is strictly increasing, $q^{*}(\theta)$ is also strictly increasing over any interval. Hence, $q^{*}(\theta)$ is strictly increasing.

Claim 3. The profits of any agent of type $\theta$ as a function of his choice of $q$ are constant in any interval in Region $I$; that is, for any $q \in\left[\alpha_{m}, \beta_{m}\right]$ with $q \geq \theta$.

The agent's profits are

$$
\left(F\left(F^{-1}\left(\frac{1}{R^{\frac{1}{n-1}}}\left(q-\alpha_{m}+F\left(\alpha_{m}\right)^{n-1} R\right)^{\frac{1}{n-1}}\right)\right)\right)^{n-1} R-(q-\theta)=F\left(\alpha_{m}\right)^{n-1} R-\left(\alpha_{m}-\theta\right) .
$$

These profits are independent of $q$.

Claim 4. The profits of any agent of type $\theta$, as a function of his choice of $q$ are decreasing in any interval in Region $C$, that is; for any $q \in\left[\beta_{m-1}, \alpha_{m}\right]$ with $q \geq \theta$.

For $q \in\left[\beta_{m-1}, \alpha_{m}\right]$ with $q \geq \theta$, the agent's profits are $F(q)^{n-1} R-(q-\theta)$. They are decreasing in $q$ since $F(q)^{n-1} R-q$ is decreasing in $q$ by construction of $\beta_{m-1}$ and $\alpha_{m}$.

Claim 5. The profits of any agent of type $\theta$ are non-increasing in his choice of $q$.

This follows from continuity and claims 3 and 4 .

Claim 6. $q^{*}(\theta)$ is a best response for an agent of type $\theta$. 
If $q^{*}(\theta)=\theta$, then the only possible change in the strategy is to increase $q$ which results, according to Claim 5, in a lower payoff. If $q^{*}(\theta)>\theta$, increasing $q$ is also non-profitable. Moreover, any decrease in $q$ for which the effort is still non-negative implies that the agent stays within the same region (recall that effort is zero in the left boundary of the region). Hence, according to Claim 3, profits remain the same.

Claims 1 to 6 imply that $q^{*}(\theta)$ is an equilibrium of the nondiscriminatory contest.

We now prove that $q^{*}(\theta)$ is the unique symmetric equilibrium. We again proceed through a series of claims. Consider any symmetric equilibrium $q(\theta)$. By arguments similar to those used in auction theory, $q(\theta)$ is necessarily continuous.

Claim 7. If $q\left(\theta^{o}\right)=\theta^{o}$ and $q\left(\theta^{o o}\right)=\theta^{o o}$ for $\theta^{o o} \geq \theta^{o}$ then $F\left(\theta^{o}\right)^{n-1} R-\theta^{o} \geq F\left(\theta^{o o}\right)^{n-1} R-$ $\theta^{o o}$.

The expected payoff of an agent of type $\theta^{\circ}$ when he chooses $q\left(\theta^{\circ}\right)=\theta^{\circ}$ is equal to $F\left(\theta^{o}\right)^{n-1} R$, which must not be less than $F\left(\theta^{o o}\right)^{n-1} R-\left(\theta^{o o}-\theta^{o}\right)$, his expected payoff if he offers quality $\theta^{o o}$. Therefore, the claim holds.

Claim 8. Consider a maximal interval $\left[\theta^{\circ}, \theta^{o o}\right]$ where $q(\theta)$ is an interior solution. Then, $q(\theta)=F(\theta)^{n-1} R+\eta$ for all $\theta \in\left[\theta^{o}, \theta^{o o}\right]$ with $\eta=\theta^{o}-F\left(\theta^{o}\right)^{n-1} R$. Moreover, either $\theta^{o o}=1$ or $\theta^{o o}$ is the first type larger than $\theta^{o}$ for which $F\left(\theta^{o o}\right)^{n-1} R-\theta^{o o}=F\left(\theta^{o}\right)^{n-1} R-\theta^{o}$ that is not a local minimum of the function $F(\theta)^{n-1} R-\theta$.

The property that $q(\theta)=F(\theta)^{n-1} R+\eta$ follows from the FOCs characterizing an interior equilibrium. To show that $\eta=\theta^{o}-F\left(\theta^{o}\right)^{n-1} R$ we distinguish between two cases.

(1) If $\theta^{\circ}>0$, then there exists an interval in Region $C$ just to the left of $\theta^{\circ}$. By continuity of $q(\theta)$, it must be the case that $q\left(\theta^{0}\right)=\theta^{0}$ which implies that $\eta=\theta^{\circ}-$ $F\left(\theta^{o}\right)^{n-1} R$.

(2) If $\theta^{o}=0$, the probability of winning is zero because the quality offered is strictly increasing in $\theta$. Hence, it cannot be that in equilibrium both agents choose $q(0)>0$, since it would lead to a negative payoff. Therefore, $F(0)^{n-1} R+\eta=0$, i.e., $\eta=0$ and $\eta=\theta^{o}-F\left(\theta^{o}\right)^{n-1} R$ holds in this case as well.

Also, by continuity, if $\theta^{o o}<1$, it must be the case that $F\left(\theta^{o o}\right)^{n-1} R+\eta=\theta^{o o}$; that is, $\theta^{o}-F\left(\theta^{o}\right)^{n-1} R=\theta^{o o}-F\left(\theta^{o o}\right)^{n-1} R$. Finally, suppose by way of contradiction, that $\theta^{o o}>\widehat{\theta}$, where $\widehat{\theta}$ is the first type which is not a local minimum of the function $F(\theta)^{n-1} R-\theta$ that satisfies $\theta^{o}-F\left(\theta^{o}\right)^{n-1} R=\widehat{\theta}-F(\widehat{\theta})^{n-1} R$. Then, $q(\theta)$ is an interior solution in an interval $[\widehat{\theta}, \widehat{\hat{\theta}}]$ where $F(\theta)^{n-1} R-\theta$ is a decreasing function. However, this is not 
possible because for $\theta \in(\widehat{\theta}, \widehat{\hat{\theta}}], q(\theta)=F(\theta)^{n-1} R+\eta=F(\theta)^{n-1} R+\theta^{o}-F\left(\theta^{o}\right)^{n-1} R=$ $F(\theta)^{n-1} R+\widehat{\theta}-F(\widehat{\theta})^{n-1} R<\theta$, since $F(\theta)^{n-1} R-\theta$ is decreasing in this interval.

Claim 9. In a maximal interval $\left[\theta^{\circ}, \theta^{o o}\right]$ where $q(\theta)=\theta$, either $\theta^{o o}=1$ or $\theta^{o o}$ is the first type such that the function $F(\theta)^{n-1} R-\theta$ is increasing on an interval $\left(\theta^{o o}, \widehat{\theta}\right]$.

Suppose, by way of contradiction, that $\theta^{o o}$ is such that the function $F(\theta)^{n-1} R-\theta$ is not increasing in an interval $\left(\theta^{o o}, \widehat{\theta}\right]$ for some $\widehat{\theta}>\theta^{o o}$. Recall that by maximality of the interval $\left[\theta^{o}, \theta^{o o}\right]$ it must be the case that $q(\theta)>\theta$ for $\theta \in\left(\theta^{o o}, \widetilde{\theta}\right]$ with $\widetilde{\theta}<\widehat{\theta}$. Hence, $q(\theta)=F(\theta)^{n-1} R+\eta=F(\theta)^{n-1} R+\theta^{o o}-F\left(\theta^{o o}\right)^{n-1} R>\theta$ for $\theta \in\left(\theta^{o o}, \tilde{\theta}\right]$. But this cannot happen if $F(\theta)^{n-1} R-\theta$ is not increasing. Furthermore, by Claim $7, \theta^{o o}$ must be the first type where this happens after $\theta^{\circ}$.

Therefore, $q^{*}(\theta)$ is the unique symmetric equilibrium, given that it is the only candidate compatible with claims 8 to 9 . Indeed, given that $F(\theta)^{n-1} R-\theta$ is decreasing for $\theta=0$, Claim 8 implies that the first interval (at equilibrium) can not be in Region $I$ because otherwise $q(\theta)<\theta$ for $\theta$ close to 0 . Once we have identified the first interval, the sequential application of claims 9 and 8 characterized the upper bounds of the following equilibrium intervals in regions $C$ and $I$. Finally, the sequence of intervals is finite because the function $F(\theta)^{n-1} R-\theta$ has a finite set of local minima.

Before we proceed to the proof of Propositions 3 and 4, and Theorems 2 and 3, we state and prove two lemmas. Lemma 1 rules out many possible strategy configurations in equilibrium for a discriminatory contest. Lemma 2 states the three strategic equilibrium configurations and the order in which they can appear.

Lemma 1 Assume the sets $\left\{\theta \in[0,1] \mid F^{\prime}(\theta)=1 / R_{A}\right\}$ and $\left\{\theta \in[0,1] \mid F^{\prime}(\theta)=1 / R_{B}\right\}$ have zero measure. Equilibrium strategies in a contest $\left(R_{A}, R_{B}\right)$ cannot give rise to a non-empty interval of qualities $\left(q_{1}, q_{2}\right) \subseteq\left[\min \left\{q_{A}(0), q_{B}(0)\right\}, q_{A}(1)\right]$ such that one of the following holds:

(a) $q_{A}^{-1}(q)<q$ and $q_{B}^{-1}(q)$ does not exist, for all $q \in\left(q_{1}, q_{2}\right)$,

(b) $q_{A}^{-1}(q)$ does not exist and $q_{B}^{-1}(q)<q$, for all $q \in\left(q_{1}, q_{2}\right)$,

(c) both $q_{A}^{-1}(q)$ and $q_{B}^{-1}(q)$ do not exist, for all $q \in\left(q_{1}, q_{2}\right)$,

(d) $q_{A}^{-1}(q)<q$ and $q_{B}^{-1}(q)=q$, for all $q \in\left(q_{1}, q_{2}\right)$,

(e) $q_{A}^{-1}(q)=q$ and $q_{B}^{-1}(q)<q$, for all $q \in\left(q_{1}, q_{2}\right)$,

(f) $q_{A}^{-1}(q)=q$ and $q_{B}^{-1}(q)$ does not exist, for all $q \in\left(q_{1}, q_{2}\right)$. 
Proof of Lemma 1. We prove the six properties by way of contradiction.

(a) If such an interval $\left(q_{1}, q_{2}\right)$ exists, then agent $A$ of type $\theta \in\left(q_{A}^{-1}\left(q_{1}\right), q_{A}^{-1}\left(q_{2}\right)\right)$ can increase his payoff by lowering the quality offered to another $q^{\prime}<q(\theta)$ such $q^{\prime} \geq$ $\max \left\{q_{1}, \theta\right\}$. This change reduces the cost and does not affect his probability of winning the contest.

(b) The proof is similar to the proof of (a).

(c) If such an interval $\left(q_{1}, q_{2}\right)$ exists, let $q_{3}=\left\{\inf q \mid q>q_{2}\right.$ and $q=q_{i}(\theta)$ for some $i=A, B$ and $\theta \in[0,1]\}$. If it is the case that $q_{3}$ is offered, that is, $q_{A}(\theta)=q_{3}$ for some $\theta \in[0,1]$ (we take agent $A$ to be the one offering $q_{3}$ without loss of generality), then $\theta<q_{3}$ (it is certainly true if $q_{2}>1$, and if $q_{2} \leq 1$ it is true since the equilibrium strategies are monotonic) and agent $A$ of type $\theta$ can increase his payoff by lowering the quality offered to another $q^{\prime}>\theta$ in the interval $\left(q_{1}, q_{2}\right)$ because this change does not affect his probability of winning the contest. By continuity, a similar argument goes through if $q_{3}$ is not reached.

(d) Suppose, by contradiction, that such an interval exists. For any type $\theta \in\left(q_{A}^{-1}\left(q_{1}\right), q_{A}^{-1}\left(q_{2}\right)\right)$, $q(\theta)$ maximizes firm $A$ 's profits $F(q) R_{A}-(q-\theta)$. Therefore, the following FOC is necessarily satisfied: $F^{\prime}\left(q_{A}(\theta)\right) R_{A}-1=0$. However, this is not possible for an interval $\left(q_{A}^{-1}\left(q_{1}\right), q_{A}^{-1}\left(q_{2}\right)\right)$ provided the set $\left\{\theta \in[0,1] / F^{\prime}(\theta)=1 / R_{A}\right\}$ has zero measure.

(e) The proof is similar to the proof of (d).

(f) Consider the maximal last interval $\left(q_{1}, q_{2}\right)$ of this type. ${ }^{30}$ Since $q_{B}(\theta) \geq q_{2}$ for every $\theta \in\left(q_{1}, q_{2}\right)$ and there cannot be a mass point, it must be the case that $q_{2}<1$. Moreover, we claim that agent $B$ must be offering quality levels arbitrarily close to $q_{2}$. Indeed, if this were not the case, the maximality of $\left(q_{1}, q_{2}\right)$ implies that agent $A$ is either reaching qualities just above $q_{2}$ by putting in positive effort or he is not reaching these qualities. The first possibility is ruled out by part (a) while the second is ruled out by part (c) of the lemma. Note that these qualities arbitrarily close to $q_{2}$ must be offered through positive effort levels by agent $B$ since they are offered by types $\theta<q_{2}$.

Given parts (b) and (e), it is necessarily the case that if $B$ puts in positive effort to reach a certain interval $\left(q_{2}, q_{3}\right), A$ also puts in positive effort to reach this interval.

Consider now the largest such $q_{3}$, we show that $\theta_{A 3} \equiv q_{A}^{-1}\left(q_{3}\right)>q_{B}^{-1}\left(q_{3}\right) \equiv \theta_{B 3}$. Notice first that $q_{2}=q_{A}^{-1}\left(q_{2}\right)>q_{B}^{-1}\left(q_{2}\right) \equiv \theta_{B 2}$ (we assume for convenience that both $q_{2}$ and $q_{3}$

\footnotetext{
${ }^{30}$ We say that $\left(q_{1}, q_{2}\right)$ is a maximal interval in a region if there does not exist an interval $\left(q_{1}^{\prime}, q_{2}^{\prime}\right)$ in that region with $q_{1}^{\prime} \leq q_{1}, q_{2}^{\prime} \geq q_{2}$ where one of the two inequalities is strict.
} 
are reached, otherwise we can make a limiting argument). Given that the qualities offered in an interior equilibrium are given by $q_{A}(\theta)=F(\theta) R_{B}+\eta_{A}$ and $q_{B}(\theta)=F(\theta) R_{A}+\eta_{B}$, $q_{3}=F\left(\theta_{A 3}\right) R_{B}+\eta_{A}=F\left(\theta_{B 3}\right) R_{A}+\eta_{B}$ and $q_{2}=F\left(q_{2}\right) R_{B}+\eta_{A}=F\left(\theta_{B 2}\right) R_{A}+\eta_{B}$. Therefore, $q_{3}-q_{2}=\left[F\left(\theta_{A 3}\right)-F\left(q_{2}\right)\right] R_{B}=\left[F\left(\theta_{B 3}\right)-F\left(\theta_{B 2}\right)\right] R_{A}$, which implies that $F\left(\theta_{A 3}\right)>F\left(\theta_{B 3}\right)-F\left(\theta_{B 2}\right)+F\left(q_{2}\right)>F\left(\theta_{B 3}\right)$, i.e., $\theta_{A 3}>\theta_{B 3}$ as we wanted to show. Therefore, there still exists another interval $\left(q_{3}, q_{4}\right)$ above $\left(q_{2}, q_{3}\right)$ where agents bid. Given that agent $B$ is putting in positive effort to reach $q_{3}$, he cannot, since there are no atoms, switch to a region of qualities that are reached by him through zero effort. Therefore, in the new interval it is again the case that $B$ does not offer any quality in it while $A$ puts in zero effort. This is the type of region we started with, in contradiction to the assumption that it is the last region of this kind. Hence, such a region cannot exist in equilibrium.

Lemma 2 (a) Consider an equilibrium $\left(q_{A}(\theta), q_{B}(\theta)\right)$ of the contest $\left(R_{A}, R_{B}\right)$ and assume the sets $\left\{\theta \in[0,1] \mid F^{\prime}(\theta)=1 / R_{A}\right\}$ and $\left\{\theta \in[0,1] \mid F^{\prime}(\theta)=1 / R_{B}\right\}$ have zero measure. Then the range of qualities offered in equilibrium $\left[q_{A}(0), q_{A}(1)\right]$ can be split into intervals, each of which belongs to either Region I, C, or J.

(b) Consider an equilibrium where there exists a (maximal) interval $\left(q_{1}, q_{2}\right)$ in Region $J$. Then, it must be followed by another interval $\left(q_{2}, q_{3}\right)$ in Region I.

(c) Consider an equilibrium where there exists a (maximal) interval $\left(q_{1}, q_{2}\right)$ in Region I. Then, it must be preceded by another interval $\left(q_{3}, q_{1}\right)$ in Region $J$.

Proof of Lemma 2. (a) It follows from Lemma 1.

(b) Consider the maximal interval $\left(q_{1}, q_{2}\right)$ in Region $J$. We notice that $q_{A}^{-1}\left(q_{2}\right) \leq q_{1}<$ 1. Therefore, there are types of agent $A$ (higher than $q_{1}$ ) that offer qualities above Region $J$. In this new interval just above $J$, agent $A$ puts in strictly positive effort. Thus, it must be the case (according to Lemma 1) that agent $B$ also puts in positive effort; that is, this new interval belongs to Region $I$.

(c) Consider the maximal interval $\left(q_{1}, q_{2}\right)$ in Region $I$. We prove this part if we show that the interval can not be preceded by an interval in Region $C$ and that it can not be the initial interval. Suppose by contradiction that either $q_{1}=0$ or that $\left(q_{1}, q_{2}\right)$ is preceded by a interval in Region $C$. In both cases, $q_{A}\left(q_{1}\right)=q_{B}\left(q_{1}\right)=q_{1}$. Given the equilibrium strategies in an interior region, $q_{A}(\theta)=F(\theta) R_{B}+q_{1}-F\left(q_{1}\right) R_{B}$ and $q_{B}(\theta)=F(\theta) R_{A}+q_{1}-F\left(q_{1}\right) R_{A}$ for any $\theta \in\left(q_{1}, q_{2}\right)$. Therefore, $q_{A}(\theta)<q_{B}(\theta)$ for any $\theta \in\left(q_{1}, q_{2}\right)$, which implies that 
$q_{B}^{-1}\left(q_{2}\right)<q_{A}^{-1}\left(q_{2}\right)$ (or that $\lim _{q \rightarrow q_{2}} q_{B}^{-1}(q)<\lim _{q \rightarrow q_{2}} q_{A}^{-1}(q)$ ). In particular, there must be an interval of qualities reached above $q_{2}$ and $q_{B}^{-1}\left(q_{2}\right)<q_{2}$. Therefore, in the interval of qualities just above $q_{2}$, agent $B$ exerts positive effort, which must be matched by agent $A$ also offering positive effort, contradicting the maximality of $\left(q_{1}, q_{2}\right)$ in Region $I$.

Proof of Proposition 3. We first prove a claim that will be used in the current proof as well as in several proofs in Section 5.

Claim 10. Suppose that agent $i$, for $i=A, B$, chooses $q_{i}(\theta)=\theta$ for all $\theta \in\left(\theta^{o}, \theta^{o o}\right)$ and that the function $F(\theta) R_{j}-\theta$, for $j \neq i$, is non-increasing in $\theta$ for $\theta \in\left(\theta^{o}, \theta^{o o}\right)$. Then, the payoff of agent $j$ of type $\theta_{j}$ is non-increasing in the quality $q$, for $q \in\left(\theta^{\circ}, \theta^{o o}\right)$ with $q \geq \theta_{j}$.

The proof of Claim 10 follows from the fact that $F(q) R_{j}-\left(q-\theta_{j}\right) \leq F\left(q^{\prime}\right) R_{j}-\left(q^{\prime}-\theta_{j}\right)$ when $q \geq q^{\prime}$ if the function $F(\theta) R_{j}-\theta$ is non-increasing between $q$ and $q^{\prime}$.

We now prove Proposition 3. If $F(\theta) R_{A}-\theta$ is non-increasing in $\theta$ for all $\theta \in[0,1]$, then the function $F(\theta) R_{B}-\theta$ is also non-increasing in $\theta$ for all $\theta \in[0,1]$ because $R_{B}<R_{A}$. Therefore, if agent $i$ chooses $q_{i}(\theta)=\theta$ for all $\theta \in[0,1]$, then agent $j \neq i$ maximizes his payoff by choosing $q_{j}(\theta)=\theta$ as well, according to Claim 10. It follows that there is an equilibrium where the agents' strategies lie in Region $C$ for all $\theta \in[0,1]$.

Moreover, if agents' equilibrium strategies lie in Region $C$ for all $\theta \in[0,1]$, then it is necessarily the case that $F(\theta) R_{A}-\theta \geq F(q) R_{A}-q$ for any $\theta \in[0,1]$ and for any $q \geq \theta$. Therefore, the function $F(\theta) R_{A}-\theta$ is non-increasing in $\theta$ for all $\theta \in[0,1]$.

Finally, suppose by contradiction that there exists another equilibrium. It must either start with an interval in Region $I_{d}$ or with an interval in Region $C$ followed by an interval in Region $I_{d}$. Therefore, there is a jump, that is, there exist two values $q_{1}$ and $q_{2}$ (where $q_{1}$ is possibly 0) with $q_{1}<q_{2}$ such that $F\left(q_{1}\right) R_{A}-q_{1} \leq F\left(q_{2}\right) R_{A}-q_{2}$. If the inequality is strict, then this contradicts the fact that the function $F(\theta) R_{A}-\theta$ is non-increasing in $\theta$. If this an equality, then $F(\theta) R_{A}-\theta$ is constant for all $\theta \in\left[q_{1}, q_{2}\right]$, which contradicts the property that the set $\left\{\theta \in[0,1] \mid F^{\prime}(\theta)=1 / R_{A}\right\}$ has zero measure.

Proof of Proposition 4. We show first that $\left(q_{A}^{I_{d}}, q_{B}^{I_{d}}\right)$ is well defined. (i) $q_{B}^{I_{d}}(\theta) \geq \theta$ for all $\theta \in[0,1]$ because $q_{B}^{I_{d}}(\theta)=\theta$ for all $\theta \in[0, \gamma)$ and $q_{B}^{I_{d}}(\theta)=F(\theta) R_{A}+\gamma-F(\gamma) R_{A} \geq \theta$ for all $\theta \geq \gamma$ according to (11). (ii) $q_{A}^{I_{d}}(\theta) \geq \theta$ for all $\theta \in[0,1]$ because $q_{A}^{I_{d}}(\theta) \geq q_{B}^{I_{d}}(\theta)$ due to the properties that $q_{A}^{I_{d}}(1)=q_{B}^{I_{d}}(1)$ and $q_{A}^{I_{d^{\prime}}}(\theta)=R_{B}<R_{A}=q_{B}^{I_{d^{\prime}}}(\theta)$.

Second, we prove a claim that will be useful at several proofs:

Claim 11. Suppose that agent $i$, for $i=A, B$, chooses $q_{i}(\theta)=F(\theta) R_{j}+\eta$ for all 
$\theta \in\left(\theta^{\circ}, \theta^{o o}\right)$, with $j \neq i$. Then, the payoff of agent $j$ of type $\theta_{j}$ is constant and equal to $\theta_{j}-\eta$ when he offers any quality $q \in\left(F\left(\theta^{o}\right) R_{j}+\eta, F\left(\theta^{o o}\right) R_{j}+\eta\right)$ with $q \geq \theta_{j}$.

Given $q_{i}(\theta)$, the payoff of agent $j$ of type $\theta_{j}$ when he offers $q \in\left(F\left(\theta^{o}\right) R_{j}+\eta, F\left(\theta^{o o}\right) R_{j}+\eta\right)$ with $q \geq \theta_{j}$ is

$$
R_{j} \operatorname{Pr}_{\theta}\left(F(\theta) R_{j}+\eta \leq q\right)-\left(q-\theta_{j}\right)=R_{j}\left(\frac{q-\eta}{R_{j}}\right)-q+\theta_{j}=\theta_{j}-\eta .
$$

Third, from Claim 11 and given agent $B$ 's strategy, the payoff of agent $A$ of type $\theta$ when he offers any quality $q \in\left[\gamma, q_{B}(1)\right]$ with $q \geq \theta$ is $\theta-\gamma+F(\gamma) R_{A}$. Similarly, the payoff of agent $B$ of type $\theta$ when he offers quality $q \in\left[\gamma, q_{B}(1)\right]$ with $q \geq \theta$ is $\theta-\gamma$, also independent of $q$. In particular, the strategies suggested are best responses one to the other for agents of type $\theta \in[\gamma, 1]$.

Agent $B$ 's payoff when offering quality $q(\theta)=\theta$ for $\theta \in[0, \gamma]$ is zero. His payoff would be negative if he were to offer any $q \in(\theta, \gamma]$ since he still has a probability zero of winning and it would be $\theta-\gamma<0$ if he were to offer any $q \in\left(\gamma, q_{B}(1)\right]$. Therefore, agent $B$ 's strategy is a best response for all $\theta \in[0, \gamma)$ as well.

Agent $A$ 's payoff when following the strategy suggested for $\theta \in[0, \gamma)$ is $\theta-\gamma+F(\gamma) R_{A}$. As shown above, his payoff is the same for any $q \geq \gamma$. If he offers $q \in[\theta, \gamma)$, then his payoff is $F(q) R_{A}-(q-\theta)$. Hence, agent $A$ 's proposed strategy is his best response if $F(q) R_{A}-(q-\theta) \leq \theta-\gamma+F(\gamma) R_{A}$ for all $q \leq \gamma$, that is, $F(q) R_{A}-q \leq F(\gamma) R_{A}-\gamma$, which is implied by (10).

We also prove that conditions (10) and (11) are necessary for $\left(q_{A}^{I_{d}}, q_{B}^{I_{d}}\right)$ to be an equilibrium. If $F(\theta) R_{A}-\theta<F(\gamma) R_{A}-\gamma$ for some $\theta>\gamma$, then $\left(q_{A}^{I_{d}}, q_{B}^{I_{d}}\right)$ cannot be an equilibrium because $q_{B}^{I_{d}}(\theta)$ would not be well defined $\left(q_{B}^{I_{d}}(\theta)<\theta\right)$. Moreover, if $F(\theta) R_{A}-\theta>F(\gamma) R_{A}-\gamma$ for some $\theta<\gamma$, then $\left(q_{A}^{I_{d}}, q_{B}^{I_{d}}\right)$ also cannot be an equilibrium because agent $A$ of type $\theta$ would strictly prefer $\theta$ to $q_{A}^{I_{d}}(\theta)$ (because his benefits under $q_{A}^{I_{d}}(\theta)$ are the same as under $\left.\gamma\right)$, contradicting the optimality of $q_{A}^{I_{d}}$.

Finally, we show that the equilibrium $\left(q_{A}^{I_{d}}, q_{B}^{I_{d}}\right)$ is unique if the inequalities are strict. We start by proving two claims:

Claim 12. Consider a maximal interval $\left[\theta_{1}, \theta_{2}\right]$ in Region $I_{d}$ and denote by $\gamma$ the cut-off corresponding to this interval. Then, $\gamma$ is increasing in both $\theta_{1}$ and $\theta_{2}$.

Indeed, the critical types $\eta_{A}, \eta_{B}$ and $\gamma$ in the interval $\left[\theta_{1}, \theta_{2}\right]$ are characterized by $q_{A}\left(\theta_{1}\right)=\gamma$, that is, $\eta_{A}=\gamma-F\left(\theta_{1}\right) R_{B} ; q_{B}(\gamma)=\gamma$, that is, $\eta_{B}=\gamma-F(\gamma) R_{A}$; and 
$q_{A}\left(\theta_{2}\right)=q_{B}\left(\theta_{2}\right)$, that is, $F\left(\theta_{2}\right) R_{B}+\gamma-F\left(\theta_{1}\right) R_{B}=F\left(\theta_{2}\right) R_{A}+\gamma-F(\gamma) R_{A}$. Therefore, $\gamma$ is characterized by $F(\gamma) R_{A}=F\left(\theta_{2}\right)\left(R_{A}-R_{B}\right)+F\left(\theta_{1}\right) R_{B}$, which implies that $\gamma$ is increasing in $\theta_{1}$ and $\theta_{2}$.

Claim 13. If $F(\theta) R_{A}-\theta$ is increasing in $\theta$ for all $\theta \in\left[\theta_{1}, \theta_{2}\right]$, then, at equilibrium, the interval $\left[\theta_{1}, \theta_{2}\right]$ is in the region $I_{d}$.

Otherwise, there must exist an interval $\left[\theta^{\circ}, \theta^{o o}\right] \subseteq\left[\theta_{1}, \theta_{2}\right]$, with $\theta^{o o}>\theta^{o}$, such that $q_{A}(\theta)=q_{B}(\theta)=\theta$ for all $\theta \in\left[\theta^{o}, \theta^{o o}\right]$. However, we claim that, for example, $q=\theta^{o}$ is not a best response for player $A$ if his type is $\theta^{\circ}$. Indeed, this player's expected profit if he chooses $q \in\left[\theta^{o}, \theta^{o o}\right]$ is $F(q) R_{A}-q+\theta^{o}$, which is increasing in $q$ if $F(\theta) R_{A}-\theta$ is increasing in $\theta$. Therefore, his expected profit is higher with, say, $q=\theta^{o o}$ than with $q=\theta^{o}$.

To prove that the equilibrium is unique, we denote by $\gamma^{*}$ the cut-off in the profile $\left(q_{A}^{I_{d}}, q_{B}^{I_{d}}\right)$, that is, $\gamma^{*} \equiv F^{-1}\left(1-\frac{R_{B}}{R_{A}}\right)$. We note that $\gamma^{*}$ is in the increasing part of the function $F(\theta) R_{A}-\theta$, according to the equations (10) and (11). Assume by way of contradiction there exists another equilibrium denoted by $E$, different from $\left(q_{A}^{I_{d}}, q_{B}^{I_{d}}\right)$. According to Claim 13, in this equilibrium $\gamma^{*}$ must still be in an interval in the Region $I_{d}$, we denote by $\Omega$ such an interval. It cannot be that $\Omega=[0,1]$ since then the two equilibria would coincide, thus, there must be an interval in Region $C$ either before or after $\gamma^{*}$. Consider first the case where there exists an interval $\left[\theta^{\circ}, \theta^{o o}\right]$ in Region $C$ before $\gamma^{*}$. If there are several such intervals, we take the one adjacent to $\Omega$ on the left, that is, $\theta^{o o}$ is the lower bound of $\Omega$. There is then a $\gamma$ (not necessarily $\gamma^{*}$ ) in $\Omega$ such that player $B$ chooses $q_{B}(\theta)=\theta$ for $\theta \in\left[\theta^{o o}, \gamma\right]$ and player $A^{\prime}$ 's profits, if of type $\theta^{o o}$, are $F\left(\theta^{o o}\right) R_{A}=F(\gamma) R_{A}-\gamma+\theta^{o o}$. Since $\theta^{o o}<\gamma^{*}$, inequality (10) implies that $F(\gamma) R_{A}-\gamma<F\left(\gamma^{*}\right) R_{A}-\gamma^{*}$ and inequality (11) implies that $\gamma<\gamma^{*}$.

If $\Omega=\left[\theta^{o o}, 1\right]$, then by Claim 12 , it must be that $\gamma>\gamma^{*}$, hence, $\Omega \neq\left[\theta^{o o}, 1\right]$ and there must exist another interval in Region $C$ with values of $\theta$ higher than $\gamma$. This, however, implies that $E$ cannot be an equilibrium. Take a particular value $\theta^{\circ o o}$ in that interval. The profit of player $A$ of type $\theta^{o o}$ in equilibrium $E$ (following $q_{A}\left(\theta^{o o}\right)=\gamma$ ) is $F(\gamma) R_{A}-\gamma+\theta^{o o}$. If he were to choose $\theta^{\circ o o}$ his profit would increase to $F\left(\theta^{\circ o o}\right) R_{A}-\theta^{o o o}+\theta^{o o}$, in contradiction to $E$ being an equilibrium. Profit increases since, $\gamma<\gamma^{*}<\theta^{o o o}$ implies (if inequalities (10) and (11) are strict) that $F(\gamma) R_{A}-\gamma<F\left(\theta^{o o o}\right) R_{A}-\theta^{o o o}$.

Second, if there is an interval $\left[\theta^{\circ}, \theta^{\circ o}\right]$ in Region $C$ after $\gamma^{*}$ but there does not exist an interval in Region $C$ before $\gamma^{*}$ then, by Claim 12, it must be that $\gamma<\gamma^{*}$. Player $A$ 's 
profit in equilibrium $E$, if of type $\theta=0$, is $F(\gamma) R_{A}-\gamma$ whereas it would be $F\left(\theta^{o}\right) R_{A}-\theta^{o}$ if it would choose $q=\theta^{\circ}$. However, $F(\gamma) R_{A}-\gamma<F\left(\theta^{\circ}\right) R_{A}-\theta^{o}$ because $\gamma<\gamma^{*}<\theta^{o}$ and strict inequalities (10) and (11), in contradiction to $q_{A}(0)=\gamma$ being a best response.

Proof of Theorem 2. (a) Given the convexity of $F(),. F^{\prime}(1) R_{A} \leq 1$ is a necessary and sufficient condition for $F(\theta) R_{A}-\theta$ to be non-increasing in $\theta$ for all $\theta \in[0,1]$. Therefore, this part follows from Proposition 3.

(b) First, we show that $\alpha$ and $\gamma$ are well defined in this region and that $\gamma>\alpha$. Equation (12) defines a function $\gamma^{1}(\alpha)$ which is increasing and such that $\gamma^{1}(1)=1$ and $\gamma^{1}(\alpha)>\alpha$ for $\alpha \in[0,1)$ (because $R_{A}>R_{B}$ ). Equation (13), together with the condition that $\gamma \geq \alpha$ defines another function $\gamma^{2}(\alpha) \cdot \gamma^{2}(\alpha)$ is defined only for values of $\alpha$ where the function $F(\theta) R_{A}-\theta$ is non-increasing, but not necessarily for all of them. Note that $\gamma^{2}(\alpha)$ is defined for all such values when $R_{A} \geq 1$; furthermore, it is certainly defined for values of $\alpha$ close enough to the minimum of the function $F(\theta) R_{A}-\theta$, which we denote $\theta_{\text {min }}$. Also note that $\gamma^{2}(\alpha)$ always lies in the increasing part of $F(\theta) R_{A}-\theta$. The function $\gamma^{2}(\alpha)$ is strictly decreasing and converges to $\theta_{\min }$ when $\alpha$ converges to $\theta_{\min }$. For this property, we use that $\theta_{\min }$ is unique. We distinguish between two cases.

When $R_{A} \in\left(\frac{1}{F^{\prime}(1)}, 1\right)$, then $F(1) R_{A}-1<0$ Therefore, there is some $\alpha^{o}$ for which $\gamma^{2}\left(\alpha^{o}\right)=1$, from which on the function is strictly decreasing until it reaches $\theta_{\min }$, where $\gamma^{2}\left(\theta_{\min }\right)=\theta_{\min }$. Given that $\gamma^{1}(\alpha)$ is strictly increasing, $\gamma^{1}(1)=1$ and $\gamma^{1}(\alpha)>\alpha$ for $\alpha \in[0,1)$, then a solution to the system of equations always exists.

When $R_{A} \in\left[1, \frac{1}{F^{\prime}(0)}\right)$, then the function $\gamma^{2}(\alpha)$ is defined for $\alpha \in\left[0, \theta_{\min }\right)$ and it takes values always lower than 1. In this case, given that $\gamma^{2}(\alpha)$ is decreasing and $\gamma^{1}(\alpha)$ is increasing, a solution exists if and only if $\gamma^{2}(0) \geq \gamma^{1}(0)$. To show that this is the case, note that $F\left(\gamma^{2}(0)\right) R_{A}-\gamma^{2}(0)=0$ and as we are in the increasing part of $F(\theta) R_{A}-\theta$ and in Region (b) where $F\left(R_{A}-R_{B}\right) R_{A}-\left(R_{A}-R_{B}\right) \leq 0$, we get that $\gamma^{2}(0) \geq R_{A}-R_{B}$. Hence, $F\left(\gamma^{2}(0)\right)=\frac{\gamma^{2}(0)}{R_{A}} \geq \frac{R_{A}-R_{B}}{R_{A}}=F\left(\gamma^{1}(0)\right)$, which implies that $\gamma^{2}(0) \geq \gamma^{1}(0)$.

Second, we show that agents' strategies are well defined, that is, the functions $\phi_{A}(\theta) \equiv$ $q_{A}^{C I_{d}}(\theta)-\theta$ and $\phi_{B}(\theta) \equiv q_{B}^{C I_{d}}(\theta)-\theta$ are non-negative for all $\theta \in[0,1]$. This trivially holds for all regions where players choose zero effort.

For $\theta \in[\gamma, 1]$ we have $q_{B}^{C I_{d}}(\theta)=F(\theta) R_{A}+\gamma-F(\gamma) R_{A}$. Given that $\gamma$ lies in the increasing part of $F(\theta) R_{A}-\theta$, we have $\phi_{B}(\theta)=F(\theta) R_{A}+\gamma-F(\gamma) R_{A}-\theta \geq 0$ for $\theta \in[\gamma, 1]$. For $\theta \in[\alpha, \gamma)$, the convexity of $\phi_{A}(\theta)=F(\theta) R_{B}+\gamma-F(\alpha) R_{B}-\theta$ implies that 
$\phi_{A}(\theta) \geq \phi_{A}(\alpha)+\phi_{A}^{\prime}(\alpha)(\theta-\alpha)=\gamma-\alpha+\left(F^{\prime}(\alpha) R_{B}-1\right)(\theta-\alpha) \geq \gamma-\alpha-(\theta-\alpha) \geq 0$. For $\theta \in[\gamma, 1]$, we note that both $\phi_{A}(\theta)$ and $\phi_{B}(\theta)$ are convex functions. Furthermore, $\phi_{A}(\gamma)>$ $\phi_{B}(\gamma)=0, \phi_{A}(1)=\phi_{B}(1)\left(\right.$ since $\left.R_{A}-F(\gamma) R_{A}=R_{B}-F(\alpha) R_{B}\right)$ and $\phi_{A}^{\prime}(\theta)<\phi_{B}^{\prime}(\theta)$ which implies that $\phi_{A}(\theta) \geq \phi_{B}(\theta)$ for all $\theta \in[\gamma, 1]$ and thus $\phi_{A}(\theta) \geq 0$ for all $\theta \in[\gamma, 1]$ as well.

Third, we prove that each agent's strategy is best response to each other.

Given agent $B$ 's strategy, the payoff of agent $A$ of type $\theta$ when he offers quality $q \in\left[\gamma, q_{B}(1)\right]$ with $q \geq \theta$ is (see Claim 11) $\theta-\gamma+F(\gamma) R_{A}$, which is independent of $q$. Similarly, the payoff of agent $B$ of type $\theta$ when offering quality $q \in\left[\gamma, q_{B}(1)\right]$ with $q \geq \theta$ is $\theta-\gamma+F(\alpha) R_{B}$, also independent of $q$. This implies, in particular, that the strategies suggested are best responses one to the other for agents of type $\theta \in[\gamma, 1]$.

The payoff of agent $B$ of type $\theta \in(\alpha, \gamma)$ is decreasing in $q$ for $q \in(\theta, \gamma)$, because no type of agent $A$ chooses qualities in $(\alpha, \gamma)$ and the payoff is constant for $q \in\left[\gamma, q_{B}(1)\right]$. Therefore, $q_{B}^{C I_{d}}(\theta)=\theta$ is a best response for all $\theta \in(\alpha, \gamma)$. The payoff of agent $B$ of type $\theta \in[0, \alpha)$ is decreasing in $q$ for $q \in[\theta, \alpha)$ because the interval $[\theta, \alpha)$ is in the decreasing part of the function $F(\theta) R_{A}-\theta$ (see Claim 10). Therefore, $q_{B}^{C I_{d}}(\theta)=\theta$ is a best response because it is first decreasing and then constant for $q \in\left[\alpha, q_{B}(1)\right]$.

Agent $A$ of type $\theta$ that chooses $q \in[0, \gamma)$, with $q \geq \theta$, obtains a payoff of $F(q) R_{A}-q+\theta$. The function $F(q) R_{A}-q$ is decreasing until $\alpha$, then it further decreases, then increases until it recovers the same value $F(\alpha) R_{A}-\alpha$ at $\gamma$ (see (13)). As we saw above, $A$ 's payoff is constant for $q \in\left[\gamma, q_{B}(1)\right]$. Therefore, $q_{A}^{C I_{d}}(\theta)=\theta$ is a best response for all $\theta \in[0, \alpha)$ and $q_{A}^{C I_{d}}(\theta)=F(\theta) R_{B}+\gamma-F(\alpha) R_{B}$ is a best response for all $\theta \in[\alpha, \gamma)$.

To show this is the unique equilibrium note that for this range of $R_{A}$, the function $F(\theta) R_{A}-\theta$ is first decreasing and then increasing. Also, if there is an interval in Region $C$ in the decreasing part of $F(\theta) R_{A}-\theta$ (such an interval cannot exist in the increasing part, according to Claim 13), then it cannot be preceded by another interval in Region $I_{d}$. Suppose otherwise. Let $\left[\theta^{\circ}, \theta^{o o}\right]$ be a maximal interval where the equilibrium lies in Region $I_{d}$ and $\left[\theta^{o o}, \theta^{o o o}\right]$ be an interval where it lies in Region $C$, both in the decreasing part of $F(\theta) R_{A}-\theta$. The strategies in $\left[\theta^{o}, \theta^{o o}\right]$ are $q_{A}(\theta)=F(\theta) R_{B}+\eta_{A}$ and $q_{B}(\theta)=F(\theta) R_{A}+\eta_{B}$, with $\eta_{B}=\theta^{o o}-F\left(\theta^{o o}\right) R_{A}$. Also, there must exist some $\gamma \in\left(\theta^{o}, \theta^{o o}\right)$ such that $F(\gamma) R_{A}+$ $\eta_{B}=\gamma$, that is, $\eta_{B}=\gamma-F(\gamma) R_{A}$. Therefore, $\theta^{o o}-F\left(\theta^{o o}\right) R_{A}=\gamma-F(\gamma) R_{A}$, which is not possible because $\gamma<\theta^{o o}$ and $F(\theta) R_{A}-\theta$ is decreasing between the two values. 
Therefore, there are only two candidate equilibria: $\left(q_{A}^{I_{d}}, q_{B}^{I_{d}}\right)$ and $\left(q_{A}^{C I_{d}}, q_{B}^{C I_{d}}\right)$. By Proposition 2, $\left(q_{A}^{I_{d}}, q_{B}^{I_{d}}\right)$ can be an equilibrium only if the inequalities of Proposition 2 are satisfied for $\gamma$ defined by $F(\gamma)=1-\frac{R_{B}}{R_{A}}$. This implies, since $F(\theta) R_{A}-\theta$ equals 0 at $\theta=0$, and is first decreasing and then increasing, that $F(\gamma) R_{A}-\gamma \geq 0$ or $R_{A}-R_{B} \geq \gamma$ or finally, $F\left(R_{A}-R_{B}\right) \geq F(\gamma)=1-\frac{R_{B}}{R_{A}}$. Each of the two conditions in this part implies this inequality is violated. $F\left(R_{A}-R_{B}\right) R_{A}-\left(R_{A}-R_{B}\right) \leq 0$ implies it directly. Also if $R_{A}<1$, then $F(\gamma) R_{A}-\gamma<0$ since $F(\gamma)<\gamma$ by convexity of $F$. Thus, the equilibrium must be of the form $C I_{d}$, the critical values of which are uniquely determined.

(c) Given the convexity of $F(\theta)$, the function $F(\theta) R_{A}-\theta$ is always non-decreasing when $R_{A} \geq \frac{1}{F^{\prime}(0)}$. Moreover, given the definition of $\gamma$, when $R_{A} \in\left[1, \frac{1}{F^{\prime}(0)}\right)$ the condition $F\left(R_{A}-R_{B}\right) R_{A}-\left(R_{A}-R_{B}\right) \geq 0$ is equivalent to $F\left(R_{A}-R_{B}\right) R_{A}-F(\gamma) R_{A} \geq 0$, or $R_{A}-R_{B} \geq \gamma$, which is equivalent to $F(\gamma) R_{A}-\gamma \geq 0$. Given that, in this region, $F(\theta) R_{A}-\theta$ is first decreasing and then increasing, if $F(\gamma) R_{A}-\gamma>0$, then inequalities (10) and (11) are strictly satisfied. Therefore, by Proposition 4 part (c) holds and the equilibrium is unique. If $F(\gamma) R_{A}-\gamma=0$, then inequalities (10) and (11) are strictly satisfied for any $\theta>0$. Similar arguments to those in the proof of Proposition 4 imply that $\left(q_{A}^{I_{d}}, q_{B}^{I_{d}}\right)$ is the unique equilibrium in this case as well.

Proof of Theorem 3. (a) This part follows from Proposition 3 because $F^{\prime}(0) R_{A} \leq 1$ and the concavity of $F$ imply that $F(\theta) R_{A}-\theta$ is non-increasing in $\theta$ for all $\theta \in[0,1]$.

(b) We first show that $\gamma$ and $\beta$ are well defined in this region and that $\beta>\gamma$. Similar to its behavior in Theorem 2, equation (15) defines a function $\beta^{2}(\gamma)$ for those values of $\gamma$ where $F(\theta) R_{A}-\theta$ is non-decreasing, but not necessarily for all of them. $\beta^{2}(\gamma)$ is defined for all such values when $R_{A} \leq 1$; furthermore it is certainly defined for values of $\gamma$ close enough to the maximum of the function $F(\theta) R_{A}-\theta$, which we denote $\theta_{\max }$. Also note that, $\beta^{2}(\gamma)$ always lies in the decreasing part of $F(\theta) R_{A}-\theta$. The function $\beta^{2}(\gamma)$ is strictly decreasing (in the interval of $\gamma$ where it is defined) and converges to $\theta_{\max }$ when $\gamma$ converges to $\theta_{\max }$. We use here the property that $\theta_{\max }$ is unique.

Equation (14) defines a function $\gamma^{1}(\beta)$. The function is increasing at least for $\beta \geq \theta_{\max }$ because $F(\theta) R_{A}-\theta$ is decreasing for $\theta \geq \theta_{\max }$ and $R_{A}>R_{B}$. Moreover, $\gamma^{1}(1)=1-R_{B}$. We distinguish between two cases. When $\beta^{2}(0)$ is well defined, that is, when $R_{A}-1 \leq 0$ then, since $R_{B}<R_{A}, \gamma^{1}(1)=1-R_{B}$ is positive. Therefore, the functions $\gamma^{1}(\beta)$ and $\beta^{2}(\gamma)$ intersect and a solution to equations (14) and (15) exists. When $R_{A} \in\left[1, \frac{1}{F^{\prime}(1)}\right]$, 
then $F(1) R_{A}-1 \leq 0$, therefore there is some $\gamma$ for which $\beta^{2}(\gamma)=1$. We denote this value by $z\left(R_{A}\right)$. The necessary and sufficient condition for (14) and (15) to intersect is that $z\left(R_{A}\right)<1-R_{B}$ or, equivalently, $F\left(1-R_{B}\right) R_{A}-\left(1-R_{B}\right)>R_{A}-1$.

Second, we show that the functions $\delta_{A}(\theta) \equiv q_{A}^{I_{d} C}(\theta)-\theta$ and $\delta_{B}(\theta) \equiv q_{B}^{I_{d} C}(\theta)-\theta$ are non-negative for all $\theta \in[0,1]$. This trivially holds if players choose zero effort.

For $\theta \in[\gamma, \beta)$, we have $F(\theta) R_{A}-\theta \geq F(\gamma) R_{A}-\gamma$ because $\gamma$ lies in the increasing part of $F(\theta) R_{A}-\theta$ and the function takes the same value for $\gamma$ and $\beta$. Hence, $\delta_{B}(\theta)=$ $F(\theta) R_{A}+\gamma-F(\gamma) R_{A}-\theta \geq 0$ for $\theta \in[\gamma, \beta)$. For $\theta \in[0, \beta), \delta_{A}(\theta)=F(\theta) R_{B}+\gamma-\theta>0$ because it is a concave function of $\theta, \delta(0)=\gamma>0$ and $\delta(\beta)=0$ by equation (14).

Third, we prove that each agent's strategy is best response to each other.

Given agent $B$ 's strategy, the payoff of agent $A$ of type $\theta \in[\beta, 1]$ is decreasing in $q$ for $q>\theta$ because the function $F(q) R_{A}-q$ is decreasing (see Claim 10); thus $q_{A}^{I_{d} C}(\theta)=\theta$ is agent $A$ 's best response. For $\theta \in[0, \beta)$, the payoff of agent $A$ is equal to $\theta+F(\gamma) R_{A}-\gamma$ for any $q \in[\gamma, \beta]$ with $q \geq \theta$ (by Claim 11) and it is decreasing for $q \in[\beta, 1]$ (by Claim 10). If agent $A$ offers quality $q \in[0, \gamma]$ with $q \geq \theta$ his payoff is $F(q) R_{A}-(q-\theta)$, which is smaller than $\theta+F(\gamma) R_{A}-\gamma$ because the function $R_{A} F(\theta)-\theta$ is increasing in that interval. Therefore, $q_{A}^{I_{d} C}(\theta)$ is an agent $A$ 's best response.

Given agent $A$ 's strategy, the payoff of agent $B$ of type $\theta \in[\beta, 1]$ when offering $q_{B}^{I_{d} C}(\theta)=\theta$ is $F(\theta) R_{B}$, which is higher than his payoff for any $q>\theta$ because we are in the decreasing part of $F(\theta) R_{A}-\theta$. The payoff of agent $B$ of type $\theta \in[\gamma, \beta]$ when offering $q_{B}^{I_{d} C}(\theta)$ is $\theta-\gamma$, which is higher than his payoff if he offers quality $q \in[\beta, 1]$ because $F(\theta) R_{B}-\theta$ is decreasing in $\theta$ for $\theta \geq \beta$. Finally, the payoff of agent $B$ of type $\theta \in[0, \gamma]$ when offering quality $\theta$ is zero. It would be negative for any $q \in[0, \gamma]$ with $q>\theta$ and, as shown above, the payoff would be first constant and then decreasing as $q$ is higher. Therefore, $q_{B}^{I_{d} C}(\theta)$ constitutes a best response to agent $A$ 's strategy.

To show that this is a unique equilibrium note that, for this range of $R_{A}$, the function $F(\theta) R_{A}-\theta$ is first increasing and then decreasing. Hence, an equilibrium must start with an interval in $I_{d}$, according to Claim 13. Moreover, if there is an interval in Region $C$ in the decreasing part of $F(\theta) R_{A}-\theta$, then it cannot be followed by another interval in Region $I_{d}$. Suppose otherwise. Let $\left[\theta^{\circ}, \theta^{o o}\right]$ be a maximal interval where the equilibrium lies in Region $C$ and $\left[\theta^{o o}, \theta^{o o o}\right]$ be an interval where it lies in Region $I_{d}$. Player $A$ 's profits must the same at $\theta^{o o}$ and at some $\gamma>\theta^{o o}$, that is (taking into account that $B$ plays 
$q_{B}(\theta)=\theta$ for all $\left.\theta \in\left[\theta^{o}, \gamma\right]\right), F\left(\theta^{o o}\right) R_{A}-\theta^{o o}=F(\gamma) R_{A}-\gamma$, which is not possible because $F(\theta) R_{A}-\theta$ is decreasing between the two values.

Therefore, there are only two candidate equilibria: $\left(q_{A}^{I_{d}}, q_{B}^{I_{d}}\right)$ and $\left(q_{A}^{I_{d} C}, q_{B}^{I_{d} C}\right) \cdot\left(q_{A}^{I_{d}}, q_{B}^{I_{d}}\right)$ can be an equilibrium only if the inequalities of Proposition 2 are satisfied for $\gamma$ such that: $F(\gamma)=1-\frac{R_{B}}{R_{A}}$. This is equivalent, since $F(\theta) R_{A}-\theta$ equals 0 at $\theta=0$, and is first increasing and then decreasing, to $F(\gamma) R_{A}-\gamma \leq R_{A}-1$ or $1-R_{B} \leq \gamma$ or finally, $F\left(1-R_{B}\right) \leq F(\gamma)=1-\frac{R_{B}}{R_{A}}$. Each of the two conditions in this part implies this inequality is violated. $F\left(1-R_{B}\right) R_{A}-\left(1-R_{B}\right)>R_{A}-1$ implies it directly. Also if $R_{A}<1$, then since $R_{A} F(\gamma)-\gamma>0$, the inequality is violated as well. Thus, the equilibrium must be of the form $I_{d} C$, the critical values of which are uniquely determined.

(c) We use Proposition 4, which we can apply directly if $R_{A} \geq \frac{1}{F^{\prime}(1)}$ because the function $F(\theta) R_{A}-\theta$ is always non-decreasing. If $R_{A} \in\left[1, \frac{1}{F^{\prime}(1)}\right)$, then conditions (10) and (11) hold if and only if $F(\gamma) R_{A}-\gamma \leq F(1) R_{A}-1$, that is, $F\left(1-R_{B}\right) R_{A}-\left(1-R_{B}\right) \leq$ $R_{A}-1$, which is the condition appearing in (c). If the inequality is strict, $\left(q_{A}^{I_{d}}, q_{B}^{I_{d}}\right)$ is the unique equilibrium according to Proposition 4. Arguments similar to those in the proof of Proposition 4 imply that the equilibrium is unique also when $F\left(1-R_{B}\right) R_{A}-\left(1-R_{B}\right)=$ $R_{A}-1$, in which case (10) and (11) are strict except for $\theta=1$.

Before we proceed to the proof of Proposition 5 we state and prove a lemma describing the designer's payoff as a function of the agents' strategies.

Lemma 3 The designer's payoff $U\left(R_{A}, R_{B}\right)$, for $R_{A} \geq R_{B}$, as a function of the agents' strategies, is the following:

(a1) If agents follow the strategy profile $\left(q_{A}^{I_{d}}, q_{B}^{I_{d}}\right)$, then

$$
U\left(R_{A}, R_{B}\right)=\frac{1}{R_{A} R_{B}} \int_{\gamma}^{R_{B}+\gamma} \pi(q)\left[2(q-\gamma)+R_{A}-R_{B}\right] d q-R_{A}+\frac{1}{2} R_{B}\left(1-\frac{R_{B}}{R_{A}}\right) .
$$

(a2) If agents follow the strategy profile $\left(q_{A}^{I_{d} C}, q_{B}^{I_{d} C}\right)$, then

$$
\begin{aligned}
U\left(R_{A}, R_{B}\right)=\frac{1}{R_{A} R_{B}} & \int_{\gamma}^{\beta} \pi(q)\left[2(q-\gamma)+F(\gamma) R_{A}\right] d q+ \\
& \quad 2 \int_{\beta}^{1} \pi(q) F(q) F^{\prime}(q) d q-\frac{1}{2}\left[R_{A}+R_{B}+F(\gamma) F(\beta)\left(R_{A}-R_{B}\right)\right]
\end{aligned}
$$


(b) If agents follow the strategy profile $\left(q_{A}^{C I_{d}}, q_{B}^{C I_{d}}\right)$, then

$$
\begin{aligned}
& U\left(R_{A}, R_{B}\right)=2 \int_{0}^{\alpha} \pi(q) F(q) F^{\prime}(q) d q+\int_{\alpha}^{\gamma} \pi(q) F(\alpha) F^{\prime}(q) d q+ \\
& \frac{1}{R_{A} R_{B}} \int_{\gamma}^{[1-F(\gamma)] R_{A}+\gamma} \pi(q)\left[2\left(q-\gamma+F(\gamma) R_{A}\right)-\left(R_{A}-R_{B}\right)\right] d q- \\
& \frac{1}{2}\left[R_{A}+R_{B}+(1-F(\alpha))^{2}\left(R_{A}-R_{B}\right)\left(1-\frac{R_{B}}{R_{A}}\right)\right] .
\end{aligned}
$$

Proof of Lemma 3. (a1) If agents follow $\left(q_{A}^{I_{d}}, q_{B}^{I_{d}}\right)$, the interval of qualities $q$ that may be offered is $\left[\gamma, R_{B}+\gamma\right]$, according to the distribution function

$$
\begin{gathered}
F^{*}(q)=\frac{1}{R_{B}}(q-\gamma) \frac{1}{R_{A}}\left[(q-\gamma)+R_{A}-R_{B}\right], \\
d F^{*}(q)=\frac{1}{R_{A} R_{B}}\left[2(q-\gamma)+R_{A}-R_{B}\right] d q .
\end{gathered}
$$

Therefore, the designer's expected income is the first part of the expression $U\left(R_{A}, R_{B}\right)$. The expected cost depends on the probability that either agent wins the contest. An agent $A$ of type $\theta$ wins the contest with probability

$$
\operatorname{Pr}_{\theta_{B}}\left(F\left(\theta_{B}\right) R_{A}+\gamma-\left(R_{A}-R_{B}\right) \leq F(\theta) R_{B}+\gamma\right)=\frac{1}{R_{A}}\left[F(\theta) R_{B}+R_{A}-R_{B}\right] .
$$

It follows that the probability that agent $A$ wins the contest is

$$
\int_{0}^{1} \frac{1}{R_{A}}\left[F(\theta) R_{B}+R_{A}-R_{B}\right] F^{\prime}(\theta) d \theta=\frac{R_{B}}{R_{A}} \frac{1}{2}\left[F(\theta)^{2}\right]_{0}^{1}+\frac{\left(R_{A}-R_{B}\right)}{R_{A}}[F(\theta)]_{0}^{1}=1-\frac{1}{2} \frac{R_{B}}{R_{A}}
$$

while the probability that $B$ wins the contest is $\frac{1}{2} \frac{R_{B}}{R_{A}}$. Therefore, the designer's expected cost is $R_{A}\left(1-\frac{1}{2} \frac{R_{B}}{R_{A}}\right)+R_{B} \frac{1}{2} \frac{R_{B}}{R_{A}}$, from which the second part of the expression $U\left(R_{A}, R_{B}\right)$ is obtained.

(a2) If agents follow the strategy profile $\left(q_{A}^{I_{d} C}, q_{B}^{I_{d} C}\right)$, the space of qualities that is reached is $[\gamma, 1]$. For $q \in[\gamma, \beta)$,

$$
\begin{gathered}
F^{*}(q)=\frac{1}{R_{B}}(q-\gamma) \frac{1}{R_{A}}\left[q-\gamma+F(\gamma) R_{A}\right], \\
d F^{*}(q)=\frac{1}{R_{A} R_{B}}\left[2(q-\gamma)+F(\gamma) R_{A}\right] d q .
\end{gathered}
$$

For $q \in[\beta, 1], d F^{*}(q)=2 F(q) F^{\prime}(q) d q$. Therefore, the expression for the designer's income follows. We compute now the probability that agent $A$ wins the contest. If his type is $\theta \in[0, \beta)$, he wins with probability

$$
\operatorname{Pr}_{\theta_{B}}\left(F\left(\theta_{B}\right) R_{A}+\gamma-F(\gamma) R_{A} \leq F(\theta) R_{B}+\gamma\right)=\frac{1}{R_{A}}\left[F(\theta) R_{B}+F(\gamma) R_{A}\right] .
$$


Moreover, agent $A$ with type $\theta \in[\beta, 1]$ wins with probability $F(\theta)$. Therefore, the probability that $A$ wins the contest is

$$
\begin{aligned}
\int_{0}^{\beta} \frac{1}{R_{A}}\left[F(\theta) R_{B}+F(\gamma) R_{A}\right] F^{\prime}(\theta) d \theta+\int_{\beta}^{1} F(\theta) F^{\prime}(\theta) d \theta= \\
\frac{R_{B}}{R_{A}} \frac{1}{2}\left[F(\theta)^{2}\right]_{0}^{\beta}+F(\gamma)[F(\theta)]_{0}^{\beta}+\frac{1}{2}\left[F(\theta)^{2}\right]_{\beta}^{1}=\frac{R_{B}}{R_{A}} \frac{1}{2} F(\beta)^{2}+F(\gamma) F(\beta)+\frac{1}{2}-\frac{1}{2} F(\beta)^{2}= \\
\frac{1}{2}+\frac{1}{2 R_{A}}\left[F(\beta) R_{B}+2 F(\gamma) R_{A}-F(\beta) R_{A}\right] F(\beta)=\frac{1}{2}+\frac{1}{2} F(\gamma) F(\beta)
\end{aligned}
$$

(where the last equality is derived from the two equations that define $\gamma$ and $\beta$ ) and the designer's expected costs are

$$
\frac{1}{2}\left[R_{A}+R_{B}+F(\gamma) F(\beta)\left(R_{A}-R_{B}\right)\right]
$$

which corresponds to the expression for the cost in part (a2) of the lemma.

(b) If agents follow $\left(q_{A}^{C I_{d}}, q_{B}^{C I_{d}}\right)$, the set of qualities that is reached is $\left[0,(1-F(\gamma)) R_{A}+\gamma\right]$. For $q \in[0, \alpha), d F^{*}(q)=2 F(q) F^{\prime}(q) d q$. For $q \in[\alpha, \gamma), F^{*}(q)=F(\alpha) F(q)$ and

$$
d F^{*}(q)=F(\alpha) F^{\prime}(q) d q
$$

Finally, for $q \in\left[\gamma,(1-F(\gamma)) R_{A}+\gamma\right]$,

$$
\begin{gathered}
F^{*}(q)=\frac{1}{R_{B}}\left(q-\gamma+F(\alpha) R_{B}\right) \frac{1}{R_{A}}\left[q-\gamma+F(\gamma) R_{A}\right], \\
d F^{*}(q)=\frac{1}{R_{A} R_{B}}\left[2(q-\gamma)+F(\alpha) R_{B}+F(\gamma) R_{A}\right] d q= \\
\frac{1}{R_{A} R_{B}}\left[2\left(q-\gamma+F(\gamma) R_{A}\right)-\left(R_{A}-R_{B}\right)\right] d q
\end{gathered}
$$

and the expression for the designer's income follows. Concerning the probability that either agent wins the contest, agent $A$ of type $\theta \in[0, \alpha)$ wins with probability $F(\theta)$ whereas, if his type is $\theta \in[\alpha, 1]$, he wins with probability

$$
\begin{aligned}
\operatorname{Pr}_{\theta_{B}}\left(F\left(\theta_{B}\right) R_{A}+\gamma-F(\gamma) R_{A} \leq F(\theta) R_{B}+\gamma-F(\alpha) R_{B}\right) & = \\
\frac{1}{R_{A}}\left[F(\theta) R_{B}-F(\alpha) R_{B}+F(\gamma) R_{A}\right] & =\frac{1}{R_{A}}\left[F(\theta) R_{B}+R_{A}-R_{B}\right] .
\end{aligned}
$$


Therefore, the probability that $A$ wins the contest is

$$
\begin{gathered}
\int_{0}^{\alpha} F(\theta) F^{\prime}(\theta) d \theta+\int_{\alpha}^{1} \frac{1}{R_{A}}\left[F(\theta) R_{B}+R_{A}-R_{B}\right] F^{\prime}(\theta) d \theta= \\
\frac{1}{2}\left[F(\theta)^{2}\right]_{0}^{\alpha}+\frac{R_{B}}{R_{A}} \frac{1}{2}\left[F(\theta)^{2}\right]_{\alpha}^{1}+\frac{\left(R_{A}-R_{B}\right)}{R_{A}}[F(\theta)]_{\alpha}^{1}= \\
1-\frac{1}{2} \frac{R_{B}}{R_{A}}+\frac{1}{2}\left(1-\frac{R_{B}}{R_{A}}\right) F(\alpha)^{2}-\left(1-\frac{R_{B}}{R_{A}}\right) F(\alpha)=\frac{1}{2}+\frac{1}{2}[1-F(\alpha)]^{2}\left(1-\frac{R_{B}}{R_{A}}\right)
\end{gathered}
$$

and the designer's expected cost is

$$
\begin{aligned}
& R_{A}\left[\frac{1}{2}+\frac{1}{2}\left(1-\frac{R_{B}}{R_{A}}\right)(1-F(\alpha))^{2}\right]+ R_{B}\left[\frac{1}{2}-\frac{1}{2}\left(1-\frac{R_{B}}{R_{A}}\right)(1-F(\alpha))^{2}\right]= \\
& \frac{1}{2}\left[R_{A}+R_{B}+(1-F(\alpha))^{2}\left(R_{A}-R_{B}\right)\left(1-\frac{R_{B}}{R_{A}}\right)\right],
\end{aligned}
$$

which corresponds to the last term of $U\left(R_{A}, R_{B}\right)$ in part (b) of the lemma.

Proof of Proposition 5. (a1) Assume the equilibrium of a nondiscriminatory contest with reward $R$ is $\left(q_{A}^{I}, q_{B}^{I}\right)$, that is, $q_{A}(\theta)=q_{B}(\theta)=F(\theta) R>\theta$ for all $\theta \in(0,1]$. Now consider a marginal change to a discriminatory contest with $R_{A}=R+\varepsilon$ and $R_{B}=$ $R-\varepsilon$. First we show that the new equilibrium is $\left(q_{A}^{I_{d}}, q_{B}^{I_{d}}\right)$.

Since $F(\theta) R-\theta$ is increasing at 0 , it is also increasing at some $\rho>0$ (with $\rho$ small enough). Moreover, $F(\theta) R-\theta$ attains a minimum $m>0$ on $[\rho, 1]$. Choose an $\varepsilon$ for which $\gamma=F^{-1}\left(1-\frac{R_{B}}{R_{A}}\right)<\rho$ and $F(\gamma)(R+\varepsilon)-\gamma<m$, which ensures that $F(\gamma)(R+\varepsilon)-\gamma<$ $F(\theta)(R+\varepsilon)-\theta$ for all $\theta \in[\rho, 1]$. Such a $\gamma$ exists because $\gamma$ goes to zero as $\varepsilon$ goes to zero. Hence the strategies given by

$$
\begin{aligned}
& q_{A}^{I_{d}}(\theta)=F(\theta) R_{B}+\gamma \text { for all } \theta \in[0,1] \\
& q_{B}^{I_{d}}(\theta)=\left\{\begin{array}{lr}
\theta & \text { for all } \theta \in[0, \gamma] \\
F(\theta) R_{A}+\gamma-F(\gamma) R_{A} & \text { for all } \theta \in(\gamma, 1]
\end{array}\right.
\end{aligned}
$$

constitute an equilibrium for the discriminatory contest by Proposition 4 .

To evaluate the optimality of the change to $R_{A}=R+\varepsilon$ and $R_{B}=R-\varepsilon$, we take the partial derivatives of the designer's payoff function $U\left(R_{A}, R_{B}\right)$ obtained in part (a1) of 
Lemma 3 with respect to $R_{A}$ and $R_{B}$.

$$
\begin{gathered}
\frac{\partial U}{\partial R_{A}}\left(R_{A}, R_{B}\right)=-\frac{1}{R_{A}^{2} R_{B}} \int_{\gamma}^{R_{B}+\gamma} \pi(q)\left[2(q-\gamma)+R_{A}-R_{B}\right] d q+ \\
\frac{1}{R_{A} R_{B}} \int_{\gamma}^{R_{B}+\gamma} \pi(q)\left[-2 \frac{\partial \gamma}{\partial R_{A}}+1\right] d q+\frac{1}{R_{A} R_{B}} \pi\left(R_{B}+\gamma\right)\left[R_{A}+R_{B}\right] \frac{\partial \gamma}{\partial R_{A}}- \\
\frac{1}{R_{A} R_{B}} \pi(\gamma)\left[R_{A}-R_{B}\right] \frac{\partial \gamma}{\partial R_{A}}-1+\frac{1}{2} \frac{R_{B}^{2}}{R_{A}^{2}}
\end{gathered}
$$

In particular, when $R_{A}=R_{B}=R$, then $\gamma=0$ and

$$
\frac{\partial U}{\partial R_{A}}\left(R_{A}=R, R_{B}=R\right)=-\frac{2}{R^{3}} \int_{0}^{R} \pi(q) q d q+\frac{1}{R^{2}}\left[1-2 \frac{\partial \gamma}{\partial R_{A}}\right] \int_{0}^{R} \pi(q) d q+\frac{2}{R^{2}} \pi(R) R \frac{\partial \gamma}{\partial R_{A}}-\frac{1}{2}
$$

Similarly,

$$
\begin{aligned}
& \frac{\partial U}{\partial R_{B}}\left(R_{A}, R_{B}\right)=-\frac{1}{R_{A} R_{B}^{2}} \int_{\gamma}^{R_{B}+\gamma} \pi(q)\left[2(q-\gamma)+R_{A}-R_{B}\right] d q+ \\
& \frac{1}{R_{A} R_{B}} \int_{\gamma}^{R_{B}+\gamma} \pi(q)\left[-2 \frac{\partial \gamma}{\partial R_{B}}-1\right] d q+\frac{1}{R_{A} R_{B}} \pi\left(R_{B}+\gamma\right)\left[R_{A}+R_{B}\right]\left[1+\frac{\partial \gamma}{\partial R_{B}}\right]- \\
& \frac{1}{R_{A} R_{B}} \pi(\gamma)\left[R_{A}-R_{B}\right] \frac{\partial \gamma}{\partial R_{B}}+\frac{1}{2}-\frac{R_{B}}{R_{A}}
\end{aligned}
$$

Therefore,

$$
\begin{array}{r}
\frac{\partial U}{\partial R_{B}}\left(R_{A}=R, R_{B}=R\right)=-\frac{2}{R^{3}} \int_{0}^{R} \pi(q) q d q+\frac{1}{R^{2}}\left[-2 \frac{\partial \gamma}{\partial R_{B}}-1\right] \int_{0}^{R} \pi(q) d q+ \\
\frac{2}{R^{2}} \pi(R) R\left[1+\frac{\partial \gamma}{\partial R_{B}}\right]-\frac{1}{2} .
\end{array}
$$

Consider now a nondiscriminatory contest $R$. If we marginally increase $R_{A}$ and simultaneously marginally decrease $R_{B}$, then the total effect is

$$
\begin{aligned}
{\left[\frac{\partial U}{\partial R_{A}}-\frac{\partial U}{\partial R_{B}}\right]\left(R_{A}=R, R_{B}=R\right) } & =\frac{2}{R^{2}}\left[1-\frac{\partial \gamma}{\partial R_{A}}+\frac{\partial \gamma}{\partial R_{B}}\right] \int_{0}^{R} \pi(q) d q- \\
\frac{2}{R^{2}} \pi(R) R\left[1-\frac{\partial \gamma}{\partial R_{A}}+\frac{\partial \gamma}{\partial R_{B}}\right] & =\frac{2}{R^{2}}\left[1-\frac{\partial \gamma}{\partial R_{A}}+\frac{\partial \gamma}{\partial R_{B}}\right]\left[\int_{0}^{R} \pi(q) d q-\pi(R) R\right]
\end{aligned}
$$

The integral $\int_{0}^{R} \pi(q) d q-\pi(R) R<0$ because $\pi(q)$ is an increasing function. Therefore, $\left[\frac{\partial U}{\partial R_{A}}-\frac{\partial U}{\partial R_{B}}\right]\left(R_{A}=R, R_{B}=R\right)>0$, that is, discriminating marginally increases the designer's payoff if and only if $1-\frac{\partial \gamma}{\partial R_{A}}+\frac{\partial \gamma}{\partial R_{B}}<0$. From $\gamma=F^{-1}\left(1-\frac{R_{B}}{R_{A}}\right)$ we have

$$
\frac{\partial \gamma}{\partial R_{A}}\left(R_{A}, R_{B}\right)=\frac{R_{B}}{R_{A}^{2}} \frac{1}{F^{\prime}(\gamma)} \text { and } \frac{\partial \gamma}{\partial R_{B}}\left(R_{A}, R_{B}\right)=-\frac{1}{R_{A}} \frac{1}{F^{\prime}(\gamma)}
$$


When we evaluate these derivatives at $R_{A}=R_{B}=R$, we obtain

$$
1-\frac{\partial \gamma}{\partial R_{A}}+\frac{\partial \gamma}{\partial R_{B}}=1-\frac{1}{R} \frac{1}{F^{\prime}(0)}-\frac{1}{R} \frac{1}{F^{\prime}(0)}=1-\frac{2}{R F^{\prime}(0)}
$$

and the result follows.

(b) As we did in part (a1), we first show that the new equilibrium is $\left(q_{A}^{C I_{d}}, q_{B}^{C I_{d}}\right)$. The nondiscriminatory equilibrium is given by

$$
q_{A}(\theta)=q_{B}(\theta)=\left\{\begin{array}{cr}
\theta & \text { for all } \theta \in\left[0, \alpha_{1}\right] \\
F(\theta) R-F\left(\alpha_{1}\right) R+\alpha_{1} & \text { for all } \theta \in\left(\alpha_{1}, 1\right]
\end{array}\right.
$$

with $F(\theta) R-\theta$ non-increasing up to $\alpha_{1}, \alpha_{1}$ is a strict local minimum and $F(\theta) R-$ $F\left(\alpha_{1}\right) R+\alpha_{1}>\theta$ for all $\theta \in\left(\alpha_{1}, 1\right]$.

Now consider $R_{A}=R+\varepsilon$ and $R_{B}=R-\varepsilon$. The function $F(\theta)(R+\varepsilon)-\theta$ obtains a strict local minimum at some $\alpha^{\prime}<\alpha_{1}$, arbitrarily close to $\alpha_{1}$ as $\varepsilon$ is very small. Given $\varepsilon$, for any $\alpha<\alpha^{\prime}$, there exists $\gamma=\gamma(\alpha)>\alpha^{\prime}$ that satisfies $F(\gamma) R_{A}-\gamma=F(\alpha) R_{A}-\alpha$. The function $\frac{\gamma(\alpha)-\alpha}{2(1-F(\alpha))}$ is positive and converges to zero as $\alpha$ tends to $\alpha^{\prime}$ because $F(\alpha)$ is bounded away from 1 . Hence, for $\varepsilon$ small enough, we can find $\alpha$ that satisfies $\frac{\gamma(\alpha)-\alpha}{2(1-F(\alpha))}=\varepsilon$. These values for $\alpha$ and $\gamma=\gamma(\alpha)$ satisfy equations (12) and (13).

By continuity, the fact that the original equilibrium was $\left(q_{A}^{C I}, q_{B}^{C I}\right)$ implies that the strategies

$$
\begin{aligned}
& q_{A}^{C I_{d}}(\theta)=\left\{\begin{array}{cc}
\theta & \text { for all } \theta \in[0, \alpha] \\
F(\theta)(R-\varepsilon)+\gamma(\alpha)-F(\alpha)(R-\varepsilon) & \text { for all } \theta \in(\alpha, 1]
\end{array}\right. \\
& q_{B}^{C I_{d}}(\theta)=\left\{\begin{array}{cc}
\theta & \text { for all } \theta \in[0, \gamma] \\
F(\theta)(R+\varepsilon)+\gamma-F(\gamma)(R-\varepsilon) & \text { for all } \theta \in(\gamma, 1]
\end{array}\right.
\end{aligned}
$$

are well defined and constitute an equilibrium.

To evaluate the optimality of the change to $R_{A}=R+\varepsilon$ and $R_{B}=R-\varepsilon$, we proceed as in part (a1).

$$
\begin{gathered}
\frac{\partial U}{\partial R_{A}}\left(R_{A}, R_{B}\right)=2 \pi(\alpha) F(\alpha) F^{\prime}(\alpha) \frac{\partial \alpha}{\partial R_{A}}+\int_{\alpha}^{\gamma} \pi(q) F^{\prime}(\alpha) F^{\prime}(q) \frac{\partial \alpha}{\partial R_{A}} d q+ \\
\pi(\gamma) F(\alpha) F^{\prime}(\gamma) \frac{\partial \gamma}{\partial R_{A}}-\pi(\alpha) F(\alpha) F^{\prime}(\alpha) \frac{\partial \alpha}{\partial R_{A}}- \\
\frac{1}{R_{A}^{2} R_{B}} \int_{\gamma}^{[1-F(\gamma)] R_{A}+\gamma} \pi(q)\left[2\left(q-\gamma+F(\gamma) R_{A}\right)-\left(R_{A}-R_{B}\right)\right] d q+ \\
\frac{1}{R_{A} R_{B}} \int_{\gamma}^{[1-F(\gamma)] R_{A}+\gamma} \pi(q)\left[2\left(-1+F^{\prime}(\gamma) R_{A}\right) \frac{\partial \gamma}{\partial R_{A}}+[2 F(\gamma)-1]\right] d q+
\end{gathered}
$$




$$
\begin{gathered}
\frac{1}{R_{A} R_{B}} I\left([1-F(\gamma)] R_{A}+\gamma\right)\left(R_{A}+R_{B}\right)\left[1-F(\gamma)+\left(1-F^{\prime}(\gamma) R_{A}\right) \frac{\partial \gamma}{\partial R_{A}}\right]- \\
\frac{1}{R_{A} R_{B}} I(\gamma)\left(2 F(\gamma) R_{A}-\left(R_{A}-R_{B}\right)\right) \frac{\partial \gamma}{\partial R_{A}}- \\
\frac{1}{2}\left[1+\left(1-\frac{R_{B}^{2}}{R_{A}^{2}}\right)(1-F(\alpha))^{2}-2\left(R_{A}-2 R_{B}+\frac{R_{B}^{2}}{R_{A}}\right)(1-F(\alpha)) F^{\prime}(\alpha) \frac{\partial \alpha}{\partial R_{A}}\right] .
\end{gathered}
$$

When $R_{A}=R_{B}=R$, then $\gamma=\alpha$ and $\alpha$ satisfies $F^{\prime}(\alpha) R=1$. Therefore,

$$
\begin{aligned}
& \frac{\partial U}{\partial R_{A}}\left(R_{A}=R, R_{B}=R\right)=\frac{1}{R} \pi(\alpha) F(\alpha) \frac{\partial \alpha}{\partial R_{A}}-\frac{1}{R} \pi(\alpha) F(\alpha) \frac{\partial \gamma}{\partial R_{A}}- \\
& \begin{aligned}
\frac{2}{R^{3}} \int_{\alpha}^{[1-F(\alpha)] R+\alpha} \pi(q)[q-\alpha+F(\alpha) R] d q+ & \frac{1}{R^{2}} \int_{\alpha}^{[1-F(\alpha)] R+\alpha} \pi(q)[2 F(\alpha)-1] d q+ \\
& \frac{2}{R} I([1-F(\widehat{\theta})] R+\alpha)[1-F(\alpha)]-\frac{1}{2} .
\end{aligned}
\end{aligned}
$$

Similar calculations show that the derivative of the designer's payoff with respect to $R_{B}$ when $R_{A}=R_{B}=R$ is

$$
\begin{aligned}
\frac{\partial U}{\partial R_{B}}\left(R_{A}=\right. & \left.R, R_{B}=R\right)=\frac{1}{R} \pi(\alpha) F(\alpha) \frac{\partial \alpha}{\partial R_{B}}-\frac{1}{R} \pi(\alpha) F(\alpha) \frac{\partial \gamma}{\partial R_{B}}- \\
& \frac{2}{R^{3}} \int_{\alpha}^{[1-F(\alpha)] R+\alpha} \pi(q)(q-\alpha+F(\alpha) R) d q+\frac{1}{R^{2}} \int_{\alpha}^{[1-F(\alpha)] R+\alpha} \pi(q) d q-\frac{1}{2} .
\end{aligned}
$$

Therefore,

$$
\begin{gathered}
{\left[\frac{\partial U}{\partial R_{A}}-\frac{\partial U}{\partial R_{B}}\right]\left(R_{A}=R, R_{B}=R\right)=\frac{1}{R} \pi(\alpha) F(\alpha)\left[\frac{\partial \alpha}{\partial R_{A}}-\frac{\partial \gamma}{\partial R_{A}}-\frac{\partial \alpha}{\partial R_{B}}+\frac{\partial \gamma}{\partial R_{B}}\right]-} \\
\frac{2}{R^{2}} \int_{\alpha}^{[1-F(\alpha)] R+\alpha} \pi(q)[1-F(\alpha)] d q+\frac{2}{R} I([1-F(\alpha)] R+\alpha)[1-F(\alpha)] .
\end{gathered}
$$

To compute the partial derivatives of $\alpha$ and $\gamma$, we use equations (12) and (13) that implicitly define these variables. Then,

$$
\left(\begin{array}{cc}
-F^{\prime}(\alpha) R_{B} & F^{\prime}(\gamma) R_{A} \\
F^{\prime}(\alpha) R_{A}-1 & -F^{\prime}(\gamma) R_{A}+1
\end{array}\right)\left(\begin{array}{c}
d \alpha \\
d \gamma
\end{array}\right)=\left(\begin{array}{cc}
1-F(\gamma) & -1+F(\alpha) \\
F(\gamma)-F(\alpha) & 0
\end{array}\right)\left(\begin{array}{c}
d R_{A} \\
d R_{B}
\end{array}\right)
$$

from which,

$$
\left(\begin{array}{c}
d \alpha \\
d \gamma
\end{array}\right)=\frac{1}{\Delta}\left(\begin{array}{cc}
-F^{\prime}(\gamma) R_{A}+1 & -F^{\prime}(\gamma) R_{A} \\
-F^{\prime}(\alpha) R_{A}+1 & -F^{\prime}(\alpha) R_{B}
\end{array}\right)\left(\begin{array}{cc}
1-F(\gamma) & -1+F(\alpha) \\
F(\gamma)-F(\alpha) & 0
\end{array}\right)\left(\begin{array}{c}
d R_{A} \\
d R_{B}
\end{array}\right)
$$

where

$$
\Delta=F^{\prime}(\alpha) R_{B}\left[F^{\prime}(\gamma) R_{A}-1\right]+F^{\prime}(\gamma) R_{A}\left[1-F^{\prime}(\alpha) R_{A}\right]
$$


We notice that $\Delta>0$ because $\alpha$ is in the decreasing part, while $\gamma$ is in the increasing part, of $F(\theta) R_{A}-\theta$, that is, $F^{\prime}(\alpha) R_{A}-1<0$ and $F^{\prime}(\gamma) R_{A}-1>0$. Therefore, $\frac{\partial \alpha}{\partial R_{A}}-\frac{\partial \gamma}{\partial R_{A}}-\frac{\partial \alpha}{\partial R_{B}}+\frac{\partial \gamma}{\partial R_{B}}=\frac{\Omega}{\Delta}$, where

$$
\begin{gathered}
\Omega=\left[-F^{\prime}(\gamma) R_{A}+1\right][1-F(\gamma)]-F^{\prime}(\gamma) R_{A}\left[F(\gamma)-F\left(\widehat{\theta}_{A}\right)\right]- \\
{\left[-F^{\prime}(\alpha) R_{A}+1\right][1-F(\gamma)]+F^{\prime}(\alpha) R_{B}[F(\gamma)-F(\alpha)]-} \\
{\left[-F^{\prime}(\gamma) R_{A}+1\right][-1+F(\alpha)]+\left[-F^{\prime}(\alpha) R_{A}+1\right][-1+F(\alpha)]=} \\
-F^{\prime}(\gamma) R_{A}+F^{\prime}(\gamma) F(\alpha) R_{A}+F^{\prime}(\alpha)[1-F(\gamma)] R_{A}+ \\
F^{\prime}(\alpha)[F(\gamma)-F(\alpha)] R_{B}+F^{\prime}(\gamma)[-1+F(\alpha)] R_{A}-F^{\prime}(\alpha)[-1+F(\alpha)] R_{A}= \\
-2 F^{\prime}(\gamma)[1-F(\alpha)] R_{A}+F^{\prime}(\alpha)\left[2 R_{A}-F(\gamma) R_{A}+F(\gamma) R_{B}-F(\alpha) R_{B}-F(\alpha) R_{A}\right]
\end{gathered}
$$

Both $\Delta$ and $\Omega$ depend on $\left(R_{A}, R_{B}\right)$ and we need to compute $\frac{\Omega}{\Delta}$ at $\left(R_{A}=R, R_{B}=R\right)$. We note that $\Omega\left(R_{A}=R, R_{B}=R\right)=0$ and $\Delta\left(R_{A}=R, R_{B}=R\right)=0$. We use that $\lim _{R_{B} \longrightarrow R_{A}} \frac{\Omega}{\Delta}\left(R_{A}, R_{B}\right)=\frac{\lim _{R_{B} \longrightarrow R_{A}} \frac{\partial \Omega}{\partial R_{B}}}{\lim _{R_{B} \longrightarrow R_{A}} \frac{\partial \Delta}{\partial R_{B}}}\left(R_{A}, R_{B}\right)$.

$$
\begin{aligned}
& \frac{\partial \Omega}{\partial R_{B}}=- 2 F^{\prime \prime}(\gamma)[1-F(\alpha)] R_{A} \frac{\partial \gamma}{\partial R_{B}}+2 F^{\prime}(\gamma) F^{\prime}(\alpha) R_{A} \frac{\partial \alpha}{\partial R_{B}}+ \\
& F^{\prime \prime}(\alpha)\left[2 R_{A}-F(\gamma) R_{A}+F(\gamma) R_{B}-F(\alpha) R_{B}-F(\alpha) R_{A}\right] \frac{\partial \alpha}{\partial R_{B}}+ \\
& F^{\prime}(\alpha)\left[F(\gamma)-F^{\prime}(\gamma)\left(R_{A}-R_{B}\right) \frac{\partial \gamma}{\partial R_{B}}-F(\alpha)-F^{\prime}(\alpha)\left(R_{B}+R_{A}\right) \frac{\partial \alpha}{\partial R_{B}}\right],
\end{aligned}
$$

which, taking into account that $\gamma=\alpha$ and $F^{\prime}(\alpha)=\frac{1}{R}$ when $R_{A}=R_{B}=R$, implies

$$
\frac{\partial \Omega}{\partial R_{B}}\left(R_{A}=R, R_{B}=R\right)=2 F^{\prime \prime}(\alpha)[1-F(\alpha)] R\left[\frac{\partial \alpha}{\partial R_{B}}-\frac{\partial \gamma}{\partial R_{B}}\right] .
$$

Similarly,

$$
\begin{aligned}
& \frac{\partial \Delta}{\partial R_{B}}=F^{\prime}(\alpha)\left[F^{\prime}(\gamma) R_{A}-1\right]+F^{\prime \prime}(\alpha) R_{B}\left[F^{\prime}(\gamma) R_{A}-1\right] \frac{\partial \alpha}{\partial R_{B}}+ \\
& F^{\prime}(\alpha) R_{B} F^{\prime \prime}(\gamma) R_{A} \frac{\partial \gamma}{\partial R_{B}}+F^{\prime \prime}(\gamma) R_{A}\left[1-F^{\prime}(\alpha) R_{A}\right] \frac{\partial \gamma}{\partial R_{B}}-F^{\prime}(\gamma) R_{A} F^{\prime \prime}(\alpha) R_{A} \frac{\partial \alpha}{\partial R_{B}}
\end{aligned}
$$

hence,

$$
\frac{\partial \Delta}{\partial R_{B}}\left(R_{A}=R, R_{B}=R\right)=F^{\prime \prime}(\alpha) R\left[\frac{\partial \gamma}{\partial R_{B}}-\frac{\partial \alpha}{\partial R_{B}}\right]
$$


We notice that $\gamma>\alpha$ as soon as $R_{A}>R_{B}$, which implies that $\frac{\partial \gamma}{\partial R_{B}}-\frac{\partial \alpha}{\partial R_{B}}>0$ at $R_{A}=R_{B}=R$. Therefore,

$$
\frac{\Omega}{\Delta}=\frac{2 F^{\prime \prime}(\alpha) R[1-F(\alpha)]\left[\frac{\partial \alpha}{\partial R_{B}}-\frac{\partial \gamma}{\partial R_{B}}\right]}{F^{\prime \prime}(\alpha) R\left[\frac{\partial \gamma}{\partial R_{B}}-\frac{\partial \alpha}{\partial R_{B}}\right]}=-2[1-F(\alpha)]
$$

We substitute $\frac{\partial \alpha}{\partial R_{A}}-\frac{\partial \gamma}{\partial R_{A}}-\frac{\partial \alpha}{\partial R_{B}}+\frac{\partial \gamma}{\partial R_{B}}$ in $\left[\frac{\partial U}{\partial R_{A}}-\frac{\partial U}{\partial R_{B}}\right]\left(R_{A}=R, R_{B}=R\right)$ to obtain

$$
\begin{aligned}
& {\left[\frac{\partial U}{\partial R_{A}}-\frac{\partial U}{\partial R_{B}}\right]\left(R_{A}=R, R_{B}=R\right)=-2 \frac{1}{R} \pi(\alpha) F(\alpha)[1-F(\alpha)]-} \\
& \frac{2}{R^{2}} \int_{\alpha}^{[1-F(\alpha)] R+\alpha} \pi(q)[1-F(\alpha)] d q+\frac{2}{R}[1-F(\alpha)] I([1-F(\alpha)] R+\alpha)=
\end{aligned}
$$

$$
\begin{array}{r}
\frac{2}{R^{2}}[1-F(\alpha)]\left[I([1-F(\alpha)] R+\alpha) R-\pi(\alpha) F(\alpha) R-\int_{\alpha}^{[1-F(\alpha)] R+\alpha} \pi(q) d q\right]> \\
\quad \frac{2}{R^{2}}[1-F(\alpha)]\left[(I([1-F(\alpha)] R+\alpha)-\pi(\alpha)) R-\int_{\alpha}^{[1-F(\alpha)] R+\alpha} \pi(q) d q\right]>
\end{array}
$$

$$
\frac{2}{R^{2}}[1-F(\alpha)]\left[(I([1-F(\alpha)] R+\alpha)-\pi(\alpha))[1-F(\alpha)] R-\int_{\alpha}^{[1-F(\alpha)] R+\alpha} \pi(q) d q\right]>0
$$

given that $\pi(q)$ is increasing. Therefore, discriminating marginally always increases the designer's payoff.

(a2) As in (a1) and (b), a marginal change in rewards leads to an $I_{d} C$ equilibrium. The marginal change in costs due to marginal discrimination is zero. Therefore, it is enough to examine the change in revenues. Rather than proceeding directly through the designer's revenue function, we examine the effect on qualities of a marginal shift from $\left(q_{A}^{I C}, q_{B}^{I C}\right)$ to $\left(q_{A}^{I_{d} C}, q_{B}^{I_{d} C}\right)$, which leads at $\varepsilon=0$ to the following derivatives:

$$
\begin{gathered}
\frac{d q_{A}^{I_{d} C}(\theta)}{d \varepsilon}=-F(\theta)+\frac{\partial \gamma}{\partial \varepsilon} \text { for all } \theta \in[0, \beta] \\
\frac{d q_{B}^{I_{d} C}(\theta)}{d \varepsilon}=F(\theta)+\frac{\partial \gamma}{\partial \varepsilon}-F^{\prime}(\gamma) R \frac{\partial \gamma}{\partial \varepsilon} \text { for all } \theta \in[0, \beta] .
\end{gathered}
$$

Differentiating equations (14) and (15) with respect to $\varepsilon$, at $\varepsilon=0$, yields

$$
\left(\begin{array}{cc}
1 & -1+F^{\prime}(\beta) R \\
1-R F^{\prime}(\beta) & -1+F^{\prime}(\beta) R
\end{array}\right)\left(\begin{array}{c}
d \gamma \\
d \beta
\end{array}\right)=\left(\begin{array}{c}
F(\beta) \\
0
\end{array}\right) d \varepsilon
$$


hence,

$$
\frac{d \gamma}{d \varepsilon}=\frac{F(\beta)}{F^{\prime}(\beta) R}>0
$$

Summing up the effect of $\varepsilon$ on both qualities, we obtain

$$
\frac{d q_{A}^{I_{d} C}(\theta)}{d \varepsilon}+\frac{d q_{B}^{I_{d} C}}{d \varepsilon}=\left[2-F^{\prime}(\beta) R\right] \frac{\partial \gamma}{\partial \varepsilon} \text { for all } \theta \in[0, \beta]
$$

whereas the effect is null for $\theta \in[\beta, 1]$, and the result follows.

Proof of Proposition 6. By Proposition 5 (b), discriminating is optimal if the equilibrium strategy profile in the nondiscriminatory contest is $\left(q_{A}^{C I}, q_{B}^{C I}\right)$. Given that $F(\theta)$ is convex and $F^{\prime}(0)=0$, the equilibrium profile is $\left(q_{A}^{C I}, q_{B}^{C I}\right)$ if the optimal $R$ satisfies $R>\frac{1}{F^{\prime}(1)}$. To show that this is the case if $v$ is large enough, we compare the profits that the designer obtains by choosing an $R>\frac{1}{F^{\prime}(1)}$ with those obtained for $R=0$ ( $R=0$ is the optimal choice among all the rewards that lead to the equilibrium profile of $\left.\left(q_{A}^{C}, q_{B}^{C}\right)\right)$. This difference in profits is equal to $v h(R)-R$, where $h(R)$ is the difference in the designer's expected income when she derives benefits $i(q)$ from an innovation, between

the case where the contestants adopt the strategy profile $\left(q_{A}^{C I_{d}}, q_{B}^{C I_{d}}\right)$ (for $\left.R>\frac{1}{F^{\prime}(1)}\right)$ and the case where the contestants adopt the strategy $\left(q_{A}^{C}, q_{B}^{C}\right)$. It is immediate that $h(R)>0$ if $i(q)$ is increasing in $q$. Therefore, $v h(R)-R>0$ if $v$ is large enough and the optimal contest necessarily implies $R>\frac{1}{F^{\prime}(1)}$.

\section{References}

[1] Amman, E., AND W. Leininger, "Asymmetric All-Pay Auctions with Incomplete Information: The Two-Player Case," Games and Economic Behavior 14(1) (1996), $1-18$.

[2] Baye, R., D. Kovenock, AND C. de Vries, "Rigging the Lobbying Process: An Application of the All-Pay Auction," American Economic Review, 83(1) (1993), 289-94.

[3] —, - AND — , "The All-Pay Auction with Complete Information," Economic Theory, 8(2) (1996), 291-305.

[4] Casas-Arce, P., AND F. Martinez-Jerez, "Handicaps in Relative Performance Compensation: An All-Pay Auction Approach," Unpublished manuscript, 2011. 
[5] Che, Y., AND I. Gale, "Optimal Design of Research Contests," American Economic Review, 93(3) (2003), 646-71.

[6] Clark, D., AND C. Riis, "Allocation Efficiency in a Competitive Bribery Game," Journal of Economic Behavior and Organization, 42(1) (2000), 109-24.

[7] Fu, Q., "A Theory of Affirmative Action in College Admissions," Economic Inquiry $44(3)$ (2006), 420-28.

[8] Fu, Q., J. Lu AND Y. Lu, "Incentivizing R\&D: Prize or Subsidies?" International Journal of Industrial Organization, 30(1) (2012), 67-79.

[9] Gurtler, O., AND M. Krakel, "Optimal Tournament Contracts for Heterogenous Workers," Journal of Economic Behavior and Organization, 75(2) (2010), 180-91.

[10] Jönsson, S., AND A. Schmutzler, "All-Pay Auctions: Implementation and Optimality," W.P. 108, University of Zurich 2013.

[11] Kawamura, K., AND I. Moreno de Barreda, "Biasing Selection Contests with Ex-ante Identical Agents," Economics Letters, 123(2) (2014), 240-43.

[12] Kaplan, T., I. Luski AND D. Wettstein, "Innovative Activity and Sunk Cost," International Journal of Industrial Organization, 21(8) (2003), 1111-33.

[13] Kirkegaard, R., "Favoritism in Asymmetric Contests: Head Starts and Handicaps," Games and Economic Behavior, 76(1) (2012), 226-48.

[14] — , "Handicaps in Incomplete Information All-Pay Auctions with a Diverse set of Bidders," European Economic Review, 64 (2013), 98-110.

[15] Konrad, K., Strategy and Dynamics in Contests, (Oxford: Oxford University Press, 2009).

[16] Kräkel, M., "On the Optimality of Unfair Tournaments," mimeo, University of Bonn 2012.

[17] Krishna, V., AND J. Morgan, "An Analysis of the War of Attrition and the All-Pay Auction," Journal of Economic Theory, 72(2) (1997), 343-62. 
[18] Levitt, S., "Optimal Incentive Schemes when Only the Agents' 'Best' Output Matters to the Principal," The Rand Journal of Economics, 26(4) (1995), 744-60.

[19] Moldovanu, B., AND A. Sela, "The Optimal Allocation of Prizes in Contests," American Economic Review, 91(3) (2001), 542-58.

[20] Newell, R. "A U.S. Innovation Strategy for Climate Change Mitigation," D.P. 200815, The Hamilton Project 2008.

[21] Newell, R., AND N. Wilson, "Technology Prizes for Climate Change Mitigation," Resources for the Future D.P. 05-33, 2005.

[22] Pérez-Castrillo, D., AND D. Wettstein, "Innovation Contests," Barcelona GSE W.P. $654,2012$.

[23] Scotchmer, S., Innovation and Incentives, (Cambridge MA: MIT Press, 2004).

[24] Seel, C., "The Value of Information in Asymmetric All-Pay Auctions," Games and Economic Behavior, 86 (2014), 330-38.

[25] Siegel, R., "All-Pay Contests," Econometrica, 77(1) (2009), 71-92.

[26] — "Asymmetric Contests with Conditional Investments," American Economic Review, 100(5) (2010), 2230-60.

[27] — "Asymmetric Contests with Interdependent Valuations," W.P. Northwestern University 2012.

[28] — "Asymmetric Contests with Head Starts and Non-Monotonic Costs," American Economic Journal: Microeconomics, 6(3) (2014), 59-105.

[29] Singh, N., AND D. Wittman, "Contests where there is Variation in the Marginal Productivity of Effort," Economic Theory, 18(3) (2001), 711-44.

[30] Winter, E., "Incentives and Discrimination," American Economic Review, 94(3) (2004), 764-73. 